\title{
DIFFUSION SEMIGROUP ON MANIFOLDS WITH TIME-DEPENDENT METRICS
}

\author{
LI-JUAN CHENG
}

\begin{abstract}
Let $L_{t}:=\Delta_{t}+Z_{t}, t \in\left[0, T_{c}\right)$ on a differential manifold equipped with a complete geometric flow $\left(g_{t}\right)_{t \in\left[0, T_{c}\right)}$, where $\Delta_{t}$ is the Laplacian operator induced by the metric $g_{t}$ and $\left(Z_{t}\right)_{t \in\left[0, T_{c}\right)}$ is a family of $C^{1, \infty}$-vector fields. In this article, we present a number of equivalent inequalities for the lower bound curvature condition, which include gradient inequalities, transportation-cost inequalities, Harnack inequalities and other functional inequalities for the semigroup associated with diffusion processes generated by $L_{t}$. To this end, we establish derivative formulae for the associated semigroup and construct couplings processes for these diffusion processes by parallel displacement and reflection.
\end{abstract}

Key words : $L_{t}$-diffusion processes, geometric flow, curvature, coupling, transportation-cost inequality, Harnack inequality, gradient inequality

MSC 2010 : 60J60, 58J65, 53C44.

\section{INTRODUCTION}

In this article, we want to clarify the connection between the behavior of distributions of diffusion processes, and the geometry of their underlying manifold carrying a geometric flow of complete Riemannian metrics, more precisely, a $d$-dimensional differential manifold $M$ equipped with a family of complete Riemannian metrics $\left(g_{t}\right)_{t \in\left[0, T_{c}\right)}$ for some $T_{c} \in(0, \infty]$, which is $C^{1}$ in $t$. Let $\nabla^{t}$ and $\Delta_{t}$ be the Levi-Civita connection and the Laplace-Beltrami operator associated with the metric $g_{t}$, respectively. For simplicity, we take the notation: for $X, Y \in T M$,

$$
\mathcal{R}_{t}^{Z}(X, Y):=\operatorname{Ric}_{t}(X, Y)-\left\langle\nabla_{X}^{t} Z_{t}, Y\right\rangle_{t}-\frac{1}{2} \partial_{t} g_{t}(X, Y),
$$

where $\mathrm{Ric}_{t}$ is the Ricci curvature tensor with respect to the metric $g_{t},\left(Z_{t}\right)_{t \in\left[0, T_{c}\right)}$ is a $C^{1, \infty}$-family of vector fields, and $\langle\cdot, \cdot\rangle_{t}:=g_{t}(\cdot, \cdot)$. Consider the diffusion process $X_{t}$ generated by $L_{t}:=\Delta_{t}+Z_{t}$ (called $L_{t}$-diffusion process $X_{t}$ ), which is assumed to be non-explosive before $T_{c}$. Let $\left\{P_{s, t}\right\}_{0 \leq s \leq t<T_{c}}$ be the semigroup associated with $X_{t}$. The main work of this article is to study behaviors of $P_{s, t}$ by using a new curvature condition, i.e. the low bound of $\mathcal{R}_{t}^{Z}$. Compared with the usual Bakry-Emery's curvature condition, it contains an additional term from the time derivative of the metric.

When the metric is independent of $t$, many excellent scholars did deep research on the development of stochastic analysis on manifolds. In [7, 18, 29], derivative formulae for the associated diffusion semigroup, known as the Bismut-Elworthy-Li formula, was given by constructing a damped gradient operator. This formula was later applied to gradient estimates, transportation-cost inequalities and other important functional inequalities, see for instance [4, 3, 5, 6, 32, 34, 38]. Besides, coupling methods play an important role on stochastic analysis. In [27], T. Lindvall and L.C.G. Rogers introduced the coupling processes for multi-dimensional diffusion processes, this idea was then extended by W.S. Kendall [22] and M. Cranston [16] to Riemannian manifolds, and further well refined in [16, 35]. It is worth mentioning that by using coupling methods, M.-F. Chen and F.-Y. Wang [8, 10, 9] gave auxiliary results for estimates of the first eigenvalue on Riemannian manifolds. Moreover, based on constructing suitable coupling processes, some equivalent important functional

Date: June 4, 2018. 
inequalities, including dimension-free Harnack inequalities, transportation-cost inequalities and gradient inequalities, were presented for the lower bound curvature condition (see e.g. [34, 38] and reference therein). For the case of manifolds with boundary, we refer the readers to [36, 37, 38] for details. All these work motivate us to extend the derivative formulae and coupling processes to the time-inhomogeneous case, which are then applied to deriving some important functional inequalities for the diffusion semigroup.

Before moving on, let us briefly recall some known results in the time-inhomogeneous Riemannian setting. In 2008, M. Arnaudon, K. Coulibaly and A. Thalmaier [1] first constructed the $g_{t}$-Brownian motions (i.e., the diffusion generated by $\frac{1}{2} \Delta_{t}$ ), and established the Bismut formula under the Ricci flow, which in particular implies gradient estimates of the associated heat semigroup, see also [15] for details. Next, by constructing horizontal diffusion processes, K. Coulibaly [2] investigated optimal transportation inequalities on time-varying manifolds. Moreover, K. Kuwada and R. Philipowski [25] studied the non-explosion of $g_{t}$-Brownian motions under some super Ricci flow. We would like to indicate that K. Kuwada [23] has developed coupling methods to estimate the gradient of the semigroup by constructing a sequence of time-inhomogeneous geodesic random walks. Very recently, R. Haslhofer and A. Naber characterize For more development on stochastic analysis in this setting, see [24] for reviewing the monotonicity of the $\mathcal{L}$-transportation cost from a probabilistic viewpoint; see [12] for stochastic analysis on the path space over time-inhomogeneous manifolds.

The paper is organized as follows. In Section 2, we will give some basic notations and notions for time-varying differential manifolds, introduce the construction of $L_{t}$-diffusion processes and then present several auxiliary results for the related diffusion semigroup.

In Section 3, we will show that, by constructing suitable local martingales, a local derivative formula can be obtained by using local geometry of the manifold. Moreover, a globe derivative formula will be studied here as well. These arguments essentially follow from A. Thalmaier [29] with some necessary modifications to our inhomogeneous context, since e.g. geometric quantities are time-dependent and the underlying process is time-inhomogeneous. As applications, we will study local gradient inequalities for the diffusion semigroup. By applying the ideas from [30], the main issue left should be to find a suitable testing function to give a subtle upper estimate.

Next, we will show a number of equivalent functional inequalities of the semigroup for the lower bound of $\mathcal{R}_{t}^{Z}$. Inspired from [3, 27], our first issue is to give some asymptotic formulae to characterize $\mathcal{R}_{t}^{Z}$. Based on these formulae and the globe derivative formula established in Theorem 3.2 , a number of equivalent gradient inequalities for the lower bound of $\mathcal{R}_{t}^{Z}$ will be given in Theorem 3.6 below. Then we will further consider dimension-free Harnack inequalities by using the gradient inequality established in Theorem 3.6. We point out that when the metric is independent of $t$, these equivalences are well-known (see e.g. [4, 3, 5, 38]). Here, we make some necessary modification as the generater operator and geometric quantities are time-dependent.

In Section 4, we will construct coupling processes for $L_{t}$-diffusion processes by solving SDEs on $M \times M$ with singular coefficients on the space-time cut-locus. Compared with [23], it looks straightforward. When the metric is independent of $t$, our construction is due to [35]. In our setting, as the SDEs we consider below are non-autonomous and the radiant process is non-differentiable on the space-time cut-locus, we need apply some results from [25, 28] and find a suitable approximation to our desired process. As applications, we will consider the transportation-cost inequality on time-inhomogeneous spaces. We would like to indicate that very recently the author uses coupling methods to investigate transportation-cost inequalities on path space of $L_{t}$-diffusion processes [13] and dimension-free Harnack inequalities on time-varying manifolds with boundary [14].

We end this section by making some conventions on the notations. Let $\mathcal{B}_{b}(M)$ be the set of all measurable functions, $C_{0}^{p}(M)$ the set of all $C^{p}$-smooth real functions with compact supports on $M$ and $C_{c}^{p}(M)$ the set of all $C^{p}$-smooth real functions with constant outside a compact set. For any two-tensor $\mathbf{T}_{t}$ and any function $f$, we write $\mathbf{T}_{t} \geq f$, if $\mathbf{T}_{t}(X, X) \geq f\langle X, X\rangle_{t}$ holds for $X \in T M$. For 
any functions $f$ and $\varphi$, respectively, defined on $\left[0, T_{c}\right) \times M$ and $\left[0, T_{c}\right) \times M \times M$, we simply write $f_{t}(x):=f(t, x)$ and $\varphi_{t}(x, y):=\varphi(t, x, y), t \in\left[0, T_{c}\right), x, y \in M . \mathbb{E}^{(s, x)}$ and $\mathbb{P}^{(s, x)}$ denote, respectively, the expectation and the probability taken for the underlying process starting from $x$ at time $s$. When $s=0$, we simply write $\mathbb{E}^{x}:=\mathbb{E}^{(0, x)}$ and $\mathbb{P}^{x}=\mathbb{P}^{(0, x)}$.

\section{Preliminaries}

Let $\mathcal{F}(M)$ be the frame bundle over $M$ and $O_{t}(M)$ the orthonormal frame bundle over $M$ with respect to the metric $g_{t}$. Define $\mathbf{p}: \mathcal{F}(M) \rightarrow M$ the projection from $\mathcal{F}(M)$ onto $M$. For any $u \in O_{t}(M)$, let $H_{X}^{t}(u)$ be the $\nabla^{t}$-horizontal lift of $X \in T_{\mathbf{p} u} M$ and $H_{i}^{t}(u)=H_{u e_{i}}^{t}(u), i=1,2, \cdots, d$, where $\left\{e_{i}\right\}_{i=1}^{d}$ is the canonical orthonormal basis of $\mathbb{R}^{d}$. Set $\left\{V_{\alpha, \beta}(u)\right\}_{\alpha, \beta=1}^{d}:=T l_{u}\left(\exp \left(E_{\alpha, \beta}\right)\right), u \in \mathcal{F}(M)$ be the canonical basis of vertical fields over $\mathcal{F}(M)$, where $E_{\alpha, \beta}$ is a canonical basis of $\mathcal{M}_{d}(\mathbb{R}), \mathcal{M}_{d}(\mathbb{R})$ is the $d \times d$ matrix space on $\mathbb{R}$, and $l_{u}: G l_{d}(\mathbb{R}) \rightarrow \mathcal{F}(M)$ is the left multiple operator from the general linear group $G l_{d}(\mathbb{R})$ to $\mathcal{F}(M)$, i.e. $l_{u} \exp \left(E_{\alpha, \beta}\right)=u \exp \left(E_{\alpha, \beta}\right)$.

Let $B_{t}:=\left(B_{t}^{1}, B_{t}^{2}, \cdots, B_{t}^{d}\right)$ be a $\mathbb{R}^{d}$-valued Brownian motion on a complete filtered probability space $\left(\Omega,\left\{\mathscr{F}_{t}\right\}_{t \geq 0}, \mathbb{P}\right)$. To construct a $L_{t}$-diffusion process, we first need to construct the corresponding horizontal diffusion process by solving the Stratonovich SDE

$$
\left\{\begin{array}{l}
d u_{t}=\sqrt{2} \sum_{i=1}^{d} H_{i}^{t}\left(u_{t}\right) \circ d B_{t}^{i}+H_{Z_{t}}^{t}\left(u_{t}\right) d t-\frac{1}{2} \sum_{\alpha, \beta=1}^{d} \mathcal{G}_{\alpha, \beta}\left(t, u_{t}\right) V_{\alpha \beta}\left(u_{t}\right) d t, \\
u_{s} \in O_{s}(M), \mathbf{p} u_{s}=x, s \in\left[0, T_{c}\right),
\end{array}\right.
$$

where $\mathcal{G}_{\alpha, \beta}\left(t, u_{t}\right):=\partial_{t} g_{t}\left(u_{t} e_{\alpha}, u_{t} e_{\beta}\right)$. Similarly as explained in [1], the last term is essential to ensure $u_{t} \in O_{t}(M)$. Since $\left\{H_{Z_{t}}^{t}\right\}_{t \in\left[0, T_{c}\right)}$ is $C^{1, \infty}$-smooth, the equation has a unique solution up to its life time $\zeta:=\lim _{n \rightarrow \infty} \zeta_{n}$, where

$$
\zeta_{n}:=\inf \left\{t \in\left[s, T_{c}\right): \rho_{t}\left(\mathbf{p} u_{0}, \mathbf{p} u_{t}\right) \geq n\right\}, n \geq 1, \quad \inf \varnothing:=T_{c},
$$

and $\rho_{t}$ stands for the Riemannian distance induced by the metric $g_{t}$. Let $X_{t}^{(s, x)}=\mathbf{p} u_{t}$. Then $X_{t}^{(s, x)}$ solves the equation

$$
d X_{t}^{(s, x)}=\sqrt{2} u_{t} \circ d B_{t}+Z_{t}\left(X_{t}^{(s, x)}\right) d t, \quad X_{s}^{(s, x)}=x:=\mathbf{p} u_{s}
$$

up to the life time $\zeta$. By the Itô formula, for any $f \in C_{0}^{2}(M)$ and $t \in\left[s, T_{c}\right)$,

$$
f\left(X_{t}^{(s, x)}\right)-f(x)-\int_{s}^{t} L_{r} f\left(X_{r}^{(s, x)}\right) d r=\sqrt{2} \int_{s}^{t}\left\langle u_{r}^{-1} \nabla^{r} f\left(X_{r}^{(s, x)}\right), d B_{r}\right\rangle
$$

is a martingale up to $\zeta$, where $\langle\cdot, \cdot\rangle$ is the inner product on $\mathbb{R}^{d}$; that is, $X_{t}^{(s, x)}$ is the diffusion process generated by $L_{t}$. When $Z_{t}=0$, the process $Y_{t}:=X_{t / 2}^{(s, x)}, t \in\left[2 s, 2 T_{c}\right)$ is generated by $\frac{1}{2} \Delta_{t / 2}$ and is known as the $g_{t / 2}$-Brownian motion. When $s=0$, we simply write $X_{t}^{(0, x)}$ as $X_{t}^{x}$ or $X_{t}$ without confusion.

Throughout this article, we assume the diffusion process $X_{t}$ generated by $L_{t}$ is non-explosive before time $T_{c}$ (see [24] for sufficient conditions to ensure the non-explosive). Then this process gives rise to an inhomogeneous Markov semigroup $\left\{P_{s, t}\right\}_{0 \leq s \leq t<T_{c}}$ on $\mathcal{B}_{b}(M)$ :

$$
P_{s, t} f(x):=\mathbb{E}\left(f\left(X_{t}^{(s, x)}\right)\right), x \in M, \quad f \in \mathcal{B}_{b}(M),
$$

which is called the diffusion semigroup generated by $L_{t}$. Here and in what follows, $\mathbb{E}$ stands for the expectation taken for the underlying process. Moreover, the Markov semigroup $\left\{P_{s, t}\right\}_{0 \leq s \leq t<T_{c}}$ enjoys the following properties.

Proposition 2.1. The following properties hold true. 
(i) For any $0 \leq s \leq t<T_{c}, f \in \mathcal{B}_{b}(M)$ and $x \in M$, there exists a unique probability measure $p_{s, t}(x, d y)$ such that

$$
P_{s, t} f(x)=\int_{M} f(y) p_{s, t}(x, d y) .
$$

(ii) The measure $p_{s, t}(x, d y)$ is equivalent to the volume measure $\mu_{t}$ with respect to the metric $g_{t}$, that is

$$
p_{s, t}(x, d y)=p(s, x ; t, y) \mu_{t}(d y)
$$

where $p(s, x ; t, y)$ is a fundamental solution to the following equation:

$$
\left\{\begin{array}{l}
\frac{\partial}{\partial s} p(\cdot, x ; t, y)(s)=-L_{s} p(s, \cdot ; t, y)(x) ; \\
\lim _{t \downarrow s} p(s, x ; t, \cdot)=\delta_{x}(\cdot) .
\end{array}\right.
$$

(iii) For any $f \in \mathcal{B}_{b}(M)$ and $0 \leq s \leq t<T_{c}$,

$$
P_{s, t} f(x)=\int_{M} f(y) p(s, x ; t, y) \mu_{t}(d y) .
$$

Moreover, the backward Kolmogorov equation

$$
\frac{d}{d s} P_{s, t} f=-L_{s} P_{s, t} f
$$

holds for all $0 \leq s \leq t<T_{c}$.

(iv) If $f \in C^{1,2}\left(\left[0, T_{c}\right) \times M\right)$ such that $\left\|\left(L_{t}+\partial_{t}\right) f\right\|_{\infty}:=\sup _{x \in M}\left|\left(L_{t}+\partial_{t}\right) f\right|(x)$ is locally bounded with respect to $t$ in $\left[0, T_{c}\right)$, then the forward Kolmogorov equation

$$
\frac{d}{d t} P_{s, t} f(t, x)=P_{s, t}\left(L_{t}+\partial_{t}\right) f(t, x)
$$

holds for all $0 \leq s \leq t<T_{c}$.

Proof. (a). Let $X_{t}$ be a $L_{t}$-diffusion process. By the Markov property, for $0 \leq s<t<T_{c}$ and $x \in M$, let $p_{s, t}(x, \cdot)=\mathcal{L}\left(X_{t} \mid X_{s}=x\right)$, the law of $X_{t}$ conditional $X_{s}=x$. Then

$$
P_{s, t} f(x)=\mathbb{E}\left(f\left(X_{t}^{(s, x)}\right)\right)=\int_{M} f(y) p_{s, t}(x, d y)
$$

for any $f \in \mathcal{B}_{b}(M)$.

(b). First, suppose $M$ is compact. Then it is easy for us to see from [19] that by replacing the Laplacian operator $\Delta_{t}$ with $\Delta_{t}+Z_{t}$, and repeating the same argument as in the proof of [19, Theorem 2.1], the existence of a fundamental solution on compact manifolds can be derived similarly. Then, for general case, given an open set $\Omega \subset M$, one can treat $\Omega$ as a manifold itself. Let us denote by $p^{\Omega}$ the heat kernel of $\Omega$. Minimality of the heat kernel implies that $p^{\Omega}$ vanishes on the boundary $\partial \Omega$, at least if $\partial \Omega$ is smooth. This implies, in turn, that $p^{\Omega}$ increases on enlarging of $\Omega$ (see [17]). The way the global heat kernel $p$ is constructed in $M$ is the following: one first defines $p^{\Omega}$ for precompact sets $\Omega$ and then lets $p:=\lim _{k \rightarrow \infty} p^{\Omega_{k}}$ where $\left\{\Omega_{k}\right\}$ is an increasing sequence of precompact open sets with smooth boundaries, which exhaust $M$. The differentiability conditions on $p(s, x ; t, y)$ follow from those on fundamental solutions in the fixed metric case, together with the $C^{1, \infty}$-smoothness of the metric.

(c). Let $u(s, x)=\int_{M} p(s, x ; t, y) f(y) \mu_{t}(d y)$. Then it is easy to see that $u$ is a solution to the following heat equation:

$$
\left\{\begin{array}{l}
\frac{\partial}{\partial s} u(s, x)=-L_{s} u(s, \cdot)(x) \\
u(t, x)=f(x)
\end{array}\right.
$$

Thus by the Feymann-Kac formula, we have

$$
\int_{M} f(y) p_{s, t}(x, d y)=P_{s, t} f(x)=u(s, x)=\int_{M} p(s, x ; t, y) f(y) \mu_{t}(d y) .
$$


(d). By using the Itô formula,

$$
f\left(t, X_{t}^{(s, x)}\right)-f(s, x)=M_{t}+\int_{s}^{t}\left(L_{r}+\partial_{r}\right) f\left(r, X_{r}^{(s, x)}\right) d r,
$$

with $M_{t}$ being a true martingale due to the boundedness of $\left(L_{r}+\partial_{r}\right) f$ on each interval $[s, t]$, we prove the result by taking expectations on both sides of the above equation.

Remark 2.2. In [19], the author proved the existence of fundamental solution by using the parametrix method introduced by E. Levi. It is easy to see from [19, 21] that the fundamental solution also exists for the equation by replacing $L=\Delta_{t}+\frac{\partial}{\partial t}$ with a locally uniformly parabolic operator on a manifolds $M$, which is written in locally coordinates as

$$
L u=\sum_{i, j} a^{i, j}(x, t) \frac{\partial^{2} u}{\partial x^{i} \partial x^{j}}+\sum_{i} b^{i}(x, t) \frac{\partial u}{\partial x^{i}}+\frac{\partial u}{\partial t},
$$

and all the coefficients involved are at least $C^{1}$.

Given $T \in\left(0, T_{c}\right)$, we deduce from (2.4) that $P_{s, T} f, s \in[0, T]$ is the solution to the following backward heat equation

$$
\left\{\begin{array}{l}
\partial_{s} u(\cdot, x)(s)=-L_{s} u(s, \cdot)(x), s \in[0, T] ; \\
u(T, x)=f(x) .
\end{array}\right.
$$

Indeed, the theory presented here is meant to be applied to our familiar forward heat equation by a time reversal. More precisely, let $\left(X_{t}^{T}\right)_{t \in[0, T]}$ be a $L_{(T-t)}$-diffusion process, which is assumed to be non-explosive before time $T$, and $\left\{\bar{P}_{s, t}\right\}_{0 \leq s \leq t \leq T}$ be the associated semigroup. Then $\bar{P}_{T-t, T} f, t \in[0, T]$ solves the equation

$$
\left\{\begin{array}{l}
\partial_{t} u(\cdot, x)(t)=L_{t} u(t, \cdot)(x), \quad t \in[0, T], \\
u(0, x)=f(x) .
\end{array}\right.
$$

We would like to indicate that in [1], derivative formulae and gradient estimates of the semigroup $\left\{\bar{P}_{s, t}\right\}_{0 \leq s \leq t \leq T}$ have been investigated by using the $g_{(T-t)}$-Brownian motion under Ricci flow.

\section{Derivative formulas AND their applications}

3.1. Derivation formulas. In this subsection, we establish derivative formulas on the local and whole manifold respectively, which is then applied to gradient estimates, dimensional-free Harnack inequalities and other functional inequalities of the semigroup.

For $u \in O_{t}(M)$, the lift operators $\mathcal{R}_{t}^{Z}(u), \mathcal{G}_{t}(u) \in \mathbb{R}^{d} \otimes \mathbb{R}^{d}$ are defined by

$$
\mathcal{R}_{t}^{Z}(u)(a, b)=\left\langle\mathcal{R}_{t}^{Z}(u) a, b\right\rangle=\mathcal{R}_{t}^{Z}(u a, u b) \text { and } \mathcal{G}_{t}(u)(a, b)=\partial_{t} g_{t}(u a, u b),
$$

where $a, b \in \mathbb{R}^{d}$. Now, let us introduce the $\mathbb{R}^{d} \otimes \mathbb{R}^{d}$-valued process $\left\{Q_{s, t}\right\}_{0<s \leq t<T_{c}}$, which solves the ODE: for $a, b \in \mathbb{R}^{d}$,

$$
\left\{\begin{array}{l}
\frac{d\left\langle Q_{s, t} a, b\right\rangle}{d t}=-\left\langle\mathcal{R}_{t}^{Z}\left(u_{t}\right) Q_{s, t} a, b\right\rangle, \\
Q_{s, s}=I,
\end{array}\right.
$$

where $u_{t}$ is the horizontal $L_{t}$-diffusion process of $X_{t}$. When $s=0$, we simply write $Q_{t}:=Q_{0, t}$.

If there exists $K \in C\left(\left[0, T_{c}\right) \times M\right)$ such that $\mathcal{R}_{t}^{Z} \geq K(t, \cdot)$ for each $t \in\left[0, T_{c}\right)$, then, from [3.1), it follows that

$$
\left\|Q_{s, t}\right\| \leq \exp \left[-\int_{s}^{t} K\left(r, X_{r}\right) d r\right]
$$

where $\|\cdot\|$ is the operator norm on $\mathbb{R}^{d}$. We now introduce a local version of derivative formula of $P_{s, t}$. When it reduces to the fixed metric case, it looks more like that given by A.Thalmaier [29]. 
Theorem 3.1. For $0 \leq s<t<T_{c}$, let $x \in M$ and $D$ be a compact domain in $[s, t] \times M$ such that $(s, x) \in D^{\circ}$, the interior of $D$. Let $\tau_{D}=\inf \left\{r \in\left(s, T_{c}\right):\left(r, X_{r}^{(s, x)}\right) \notin D\right\}$ and $F \in C^{1,2}([s, t] \times D)$ satisfy the heat equation

$$
\partial_{r} F(\cdot, x)(r)=-L_{r} F(r, \cdot)(x),
$$

for all $(r, x) \in[s, t] \times D$. Then for any adapted absolutely continuous $\mathbb{R}_{+}$-valued process $h$ such that $h(s)=0$ and $h(r)=1$ for all $r \geq t \wedge \tau_{D}$, and $\mathbb{E}^{(s, x)}\left(\int_{s}^{t} h^{\prime}(r)^{2} d r\right)^{\alpha}<\infty$ for some $\alpha>\frac{1}{2}$, we have

$$
\left(u_{s}\right)^{-1} \nabla^{s} F(s, \cdot)(x)=\frac{1}{\sqrt{2}} \mathbb{E}^{(s, x)}\left\{F\left(t \wedge \tau_{D}, X_{t \wedge \tau_{D}}\right) \int_{s}^{t} h^{\prime}(r) Q_{s, r}^{*} d B_{r}\right\},
$$

where $Q_{s, r}^{*}$ is the transpose of $Q_{s, r}$.

Proof. Without loss of generality, we assume $s=0$ and simply drop the upper script $x$ in $X_{t}^{x}$. Since $Z$ is a $C^{1, \infty}$ vector field and $F$ is a classic solution of $(3.2)$, then it is easy to see that $F \in C^{1,3}([s, t] \times D)$. For $u \in O_{r}(M)$ and $r \in\left[0, T_{c}\right)$, let $G(u, r)=u^{-1} \nabla^{r} F_{r}(\mathbf{p} u)$. Then according to (3.2), we have

$$
\frac{\partial}{\partial r} G(u, r)=\sum_{i=1}^{d} \frac{\partial}{\partial r} u e_{i} F_{r}(\mathbf{p} u) e_{i}=-\sum_{i=1}^{d} u e_{i}\left(L_{r} F_{r}\right)(\mathbf{p} u) e_{i}=-u^{-1} \nabla^{r} L_{r} F_{r}(\mathbf{p} u) .
$$

By this and the Bochner-Weitzenböck formula, we see that for $r \in[0, t]$,

$$
\frac{\partial}{\partial r} G(u, r)=-L_{O_{r}(M)} G(\cdot, r)(u)+\left(\mathcal{R}_{r}^{Z}(u)+\frac{1}{2} \mathcal{G}_{r}(u)\right) G(u, r),
$$

where $L_{O_{r}(M)}:=\Delta_{O_{r}(M)}+H_{Z_{r}}^{r}$ and $\Delta_{O_{r}(M)}$ is the horizontal Laplacian operator of $\Delta_{r}$. On the other hand, noting that $u_{r}$ is the solution to (2.1), and using the Itô formula, one obtains that for fixed $t_{0} \in[0, t]$,

$$
d G\left(u_{r}, t_{0}\right)=d M_{r}+L_{O_{r}(M)} G\left(\cdot, t_{0}\right)\left(u_{r}\right) d r-\frac{1}{2} \sum_{\alpha, \beta} \mathcal{G}_{\alpha, \beta}\left(t_{0}, u_{r}\right) V_{\alpha, \beta}\left(u_{r}\right) G\left(\cdot, t_{0}\right)\left(u_{r}\right) d r,
$$

where $d M_{r}:=\sqrt{2} H_{u_{r} d B_{r}}^{r} G\left(\cdot, t_{0}\right)\left(u_{r}\right)$. Moreover, the last term above on the rightmost hand satisfies that

$$
\begin{aligned}
-\frac{1}{2} \sum_{\alpha, \beta} \mathcal{G}_{\alpha, \beta}\left(t_{0}, u_{r}\right) V_{\alpha, \beta}\left(u_{r}\right) G\left(\cdot, t_{0}\right)\left(u_{r}\right) \\
=-\frac{1}{2} \sum_{\alpha, \beta} \mathcal{G}_{\alpha, \beta}\left(t_{0}, u_{r}\right)\left(u_{r} e_{\beta} F_{r}\right)\left(X_{r}\right) \cdot e_{\alpha} \\
=-\frac{1}{2} \mathcal{G}_{t_{0}}\left(u_{r}\right) G\left(u_{r}, t_{0}\right) .
\end{aligned}
$$

With this, (3.5) and (3.4), we conclude that

$$
d\left\langle\nabla^{r} F_{r}\left(X_{r}\right), u_{r} Q_{r} a\right\rangle_{r}=d\left\langle G\left(u_{r}, r\right), Q_{r} a\right\rangle=\sqrt{2} \operatorname{Hess}_{F_{r}}^{r}\left(u_{r} \mathrm{~d} B_{r}, u_{r} Q_{r} a\right)\left(X_{r}\right),
$$

which implies that $\left\langle\nabla^{r} F_{r}\left(X_{r}\right), u_{r} Q_{r} a\right\rangle_{r}$ is a local martingale.

On the other hand, by the Itô formula, one has

$$
d F\left(r, X_{r}\right)=\sqrt{2}\left\langle\nabla^{r} F_{r}\left(X_{r}\right), u_{r} d B_{r}\right\rangle_{r},
$$

and

$$
F\left(t \wedge \tau_{D}, X_{t \wedge \tau_{D}}\right)=F(0, x)+\sqrt{2} \int_{0}^{t \wedge \tau_{D}}\left\langle\nabla^{r} F_{r}\left(X_{r}\right), u_{r} d B_{r}\right\rangle_{r}
$$


Therefore, noting that $h^{\prime}(r)=0$ for $r \geq t \wedge \tau_{D}$, we have

$$
\begin{aligned}
& \mathbb{E}^{x}\left\{F\left(t \wedge \tau_{D}, X_{t \wedge \tau_{D}}\right) \int_{0}^{t}\left\langle h^{\prime}(r) Q_{r} a, d B_{r}\right\rangle\right\} \\
= & \mathbb{E}^{x}\left\{\left(F(0, x)+\sqrt{2} \int_{0}^{t \wedge \tau_{D}}\left\langle\nabla^{r} F_{r}\left(X_{r}\right), u_{r} d B_{r}\right\rangle_{r}\right) \int_{0}^{t}\left\langle h^{\prime}(r) Q_{r} a, d B_{r}\right\rangle\right\} .
\end{aligned}
$$

As $\int_{0}^{t}\left\langle h^{\prime}(r) Q_{r} a, d B_{r}\right\rangle$ is a true martingale, we then have

$$
\begin{aligned}
& \frac{1}{\sqrt{2}} \mathbb{E}^{x}\left\{F\left(t \wedge \tau_{D}, X_{t \wedge \tau_{D}}\right) \int_{0}^{t}\left\langle h^{\prime}(r) Q_{r} a, d B_{r}\right\rangle\right\} \\
= & \mathbb{E}^{x}\left\{\int_{0}^{t}\left\langle\nabla^{r} F_{r}\left(X_{r}\right), u_{r} Q_{r} a\right\rangle_{r}(h-1)^{\prime}(r) d r\right\} \\
= & \mathbb{E}^{x}\left\{\left.\left[\left\langle\nabla^{r} F_{r}\left(X_{r}\right), u_{r} Q_{r} a\right\rangle_{r} \cdot(h-1)(r)\right]\right|_{0} ^{t}\right\}-\mathbb{E}^{x} \int_{0}^{t}(h-1)(r) d\left\langle\nabla^{r} F_{r}\left(X_{r}\right), u_{r} Q_{r} a\right\rangle_{r} \\
= & \left\langle\nabla^{0} F_{0}(x), u_{0} a\right\rangle_{0},
\end{aligned}
$$

where the last step follows from (3.6) that $\int_{0}^{t}(h-1)(r) d\left\langle\nabla^{r} F_{r}\left(X_{r}\right), u_{r} Q_{r} a\right\rangle_{r}$ is a true martingale. Now given arbitrary $s \in\left[0, T_{c}\right.$ ), repeating the above argument, we prove (3.3) and then finish the proof.

Next, we introduce the following globe derivative formula for $P_{s, t}$ without using hitting time. For the corresponding result in the fixed metric case, we refer the readers to [30]. Let $\operatorname{Cut}_{t}(x)$ be the set of the $g_{t}$-cut-locus of $x$ on $M$.

Theorem 3.2. Assume that for each $s \in\left[0, T_{c}\right),\left(L_{s}+\partial_{s}\right) \rho_{s}^{2} \leq c+h_{1}(s)+h_{2}(s) \rho_{s}^{2}$ holds outside $\mathrm{Cut}_{s}(o)$ for some constant $c>0$ and some non-negative functions $h_{1}, h_{2} \in C\left(\left[0, T_{c}\right)\right)$. If

$$
\mathcal{R}_{s}^{Z} \geq h_{3}(s)-16 e^{-\int_{0}^{s}\left(h_{2}(r)+16\right) d r} \rho_{s}^{2}
$$

holds for some $h_{3} \in C\left(\left[0, T_{c}\right)\right)$, then for all $0 \leq s \leq t<T_{c}$ and $h \in C^{1}([s, t])$ satisfying $h(s)=0$ and $h(t)=1$,

$$
u_{s}^{-1} \nabla^{s} P_{s, t} f(x)=\mathbb{E}^{(s, x)}\left\{Q_{s, t}^{*} u_{t}^{-1} \nabla^{t} f\left(X_{t}\right)\right\}=\frac{1}{\sqrt{2}} \mathbb{E}^{(s, x)}\left\{f\left(X_{t}\right) \int_{s}^{t} h^{\prime}(r) Q_{s, r}^{*} d B_{r}\right\},
$$

where $x \in M$ and $f \in C^{1}(M)$ such that $f$ is constant outside a compact set. In particular, by taking $h(r)=\frac{(r-s) \wedge(t-s)}{t-s}$, it holds

$$
u_{s}^{-1} \nabla^{s} P_{s, t} f(x)=\frac{1}{\sqrt{2}(t-s)} \mathbb{E}^{(s, x)}\left\{f\left(X_{t}\right) \int_{s}^{t} Q_{s, r}^{*} d B_{r}\right\} .
$$

Proof. We again assume $s=0$. By the Itô formula (see [25, Theorem 2]),

$$
d \rho_{r}^{2}\left(X_{r}\right) \leq 2 \sqrt{2} \rho_{r}\left(X_{r}\right) d b_{r}+\left(c+h_{1}(r)+h_{2}(r) \rho_{r}^{2}\left(X_{r}\right)\right) d r
$$

holds for some one-dimensional Brownian motion $b_{t}$. Let $\lambda(r)=\int_{0}^{r}\left(h_{2}(s)+16\right) d s$. Then, we have

$$
\begin{aligned}
d\left[e^{-\lambda(r)} \rho_{r}^{2}\left(X_{r}\right)\right] \leq & e^{-\lambda(r)}\left[2 \sqrt{2} \rho_{r}\left(X_{r}\right) d b_{r}+\left(c+h_{1}(r)+h_{2}(r) \rho_{r}^{2}\left(X_{r}\right)\right) d r\right] \\
& -e^{-\lambda(r)}\left(h_{2}(r)+16\right) \rho_{r}^{2}\left(X_{r}\right) d r \\
= & 2 \sqrt{2} e^{-\lambda(r)} \rho_{r}\left(X_{r}\right) d b_{r}-16 e^{-\lambda(r)} \rho_{r}^{2}\left(X_{r}\right) d r+\left(c+h_{1}(r)\right) e^{-\lambda(r)} d r .
\end{aligned}
$$


Thus, letting $C(t, x)=e^{\rho_{0}^{2}(x)+c t+\int_{0}^{t} h_{1}(s) d s}$, we obtain

$$
\begin{aligned}
\mathbb{E}^{x} \exp \left\{16 \int_{0}^{t \wedge \zeta_{n}} \rho_{r}^{2}\left(X_{r}\right) e^{-\lambda(r)} d r\right\} & \leq \mathbb{E}^{x} \exp \left\{2 \sqrt{2} \int_{0}^{t \wedge \zeta_{n}} \rho_{r}\left(X_{r}\right) e^{-\lambda(r)} d b_{r}\right\} \cdot C(t, x) \\
& \leq \mathbb{E}^{x} \exp \left\{16 \int_{0}^{t \wedge \zeta_{n}} \rho_{r}^{2}\left(X_{r}\right) e^{-2 \lambda(r)} d r\right\}^{1 / 2} \cdot C(t, x),
\end{aligned}
$$

where $\zeta_{n}$ is defined as in (2.2) with $s=0$. From this, it is easy to deduce that

$$
\mathbb{E}^{x} \exp \left\{16 \int_{0}^{t \wedge \zeta_{n}} \rho_{r}^{2}\left(X_{r}\right) e^{-\lambda(r)} d r\right\} \leq C(t, x)^{2} .
$$

Now letting $n \rightarrow \infty$, we arrive at

$$
\mathbb{E}^{x} \exp \left\{16 \int_{0}^{t} \rho_{r}^{2}\left(X_{r}\right) e^{-\lambda(r)} d r\right\} \leq C(t, x)^{2} .
$$

Combining this with (3.8), and letting $K(r, x):=h_{3}(r)-16 e^{-\int_{0}^{r}\left(h_{2}(s)+16\right) d s} \rho_{r}^{2}$, we deduce that $\mathcal{R}_{r}^{Z} \geq$ $K_{r}, r \in[0, t]$, and

$$
\sup _{x \in \mathbf{K}} \mathbb{E}^{x} e^{\int_{0}^{t} K^{-}\left(r, X_{r}\right) d r} \leq \sup _{x \in \mathbf{K}} \mathbb{E}^{x} \exp \left\{16 \int_{0}^{t} e^{-\int_{0}^{r}\left(h_{2}(u)+16\right) d u} \rho_{r}^{2}\left(X_{r}\right) d r\right\}<\infty,
$$

where $\mathbf{K} \subset M$ is a compact subset. Then, following the proof of [26, Theorems 8.5 and 9.1], it is easy to derive from (3.10) that $\sup _{r \in[0, t]}\left|\left\|\left.\nabla^{r} P_{r, t} f\right|_{r}\right\|_{\infty}<\infty\right.$. Therefore, the first equality in (3.9) for $s=0$ follows from (3.6) by taking $F(r, x)=P_{r, t} f(x), r \in[0, t]$.

Next, due to the first equality in (3.9) for $s=0$, we know that for any $a \in \mathbb{R}^{d}$ with $\|a\|=1$,

$$
\mathbb{E}^{x} \sup _{r \in[0, t]}\left|\left\langle\nabla^{r} P_{r, t} f\left(X_{r}\right), u_{r} Q_{r} a\right\rangle_{r}\right| \leq \sup _{r \in[0, t]}\left\|\left|\nabla^{r} P_{r, t} f\right|_{r}\right\|_{\infty} \mathbb{E}^{x} e^{\int_{0}^{t} K^{-}\left(r, X_{r}\right) d r}<\infty,
$$

which implies that

$$
\left\langle\nabla^{r} P_{r, t} f\left(X_{r}\right), u_{r} Q_{r} a\right\rangle_{r}, r \in[0, t]
$$

is a uniformly integrable martingale. Hence (3.3) holds for $t$ in place of $t \wedge \tau_{D}$ and any $h \in C^{1}([0, t])$ with $h(0)=0$ and $h(t)=1$. Therefore, the second equality in (3.9) holds for $s=0$.

If there exists a non-negative $\phi \in C([0, \infty))$ and $h \in C\left(\left[0, T_{c}\right)\right)$ such that $\mathcal{R}_{t}^{Z} \geq-h(t) \phi\left(\rho_{t}\right)$, then by a similar argument as in the proof of [25, Lemma 9], there exists a non-increasing function $F$ satisfying $\lim _{r \rightarrow 0} r F(r)<\infty$ such that

$$
\left(L_{t}+\partial_{t}\right) \rho_{t}(x) \leq F\left(\rho_{t}(x)\right)+h(t) \int_{0}^{\rho_{t}(x)} \phi(s) d s+\left|Z_{t}(o)\right|_{t} .
$$

Hence, the assumptions in Theorem 3.2 are ensured by each of the following conditions:

(A1) there exists a non-negative function $C \in C\left(\left[0, T_{c}\right)\right)$ such that $\mathcal{R}_{t}^{Z} \geq-C(t)$ for all $t \in\left[0, T_{c}\right)$;

(A2) there exist two non-negative functions $C_{1}, C_{2} \in C\left(\left[0, T_{c}\right)\right)$, such that for all $t \in\left[0, T_{c}\right)$,

$$
\operatorname{Ric}_{t} \geq-C_{1}(t)\left(1+\rho_{t}^{2}\right) \text { and } \partial_{t} \rho_{t}+\left\langle Z_{t}, \nabla^{t} \rho_{t}\right\rangle_{t} \leq C_{2}(t)\left(1+\rho_{t}\right)
$$

Remark 3.3. If (A2) holds, then

$$
\begin{aligned}
\left(L_{t}+\partial_{t}\right) \rho_{t}^{2} & =2 \rho_{t}\left(L_{t}+\partial_{t}\right) \rho_{t}+2=2 \rho_{t}\left(\Delta_{t}+\partial_{t}+Z_{t}\right) \rho_{t}+2 \\
& \leq 2 \rho_{t} \Delta_{t} \rho_{t}+2 C_{2}(t)\left(\rho_{t}+\rho_{t}^{2}\right)+2 \\
& \leq 2 \rho_{t} \sqrt{(d-1) C_{1}(t)\left(1+\rho_{t}^{2}\right)} \operatorname{coth}\left(\sqrt{C_{1}(t)\left(1+\rho_{t}^{2}\right) /(d-1)} \rho_{t}\right)+2 C_{2}(t)\left(\rho_{t}+\rho_{t}^{2}\right)+2 .
\end{aligned}
$$

Combining this with the inequality $\operatorname{coth}(s) \leq 1+s^{-1}$, we know that under (A2), the conditions in Theorem 3.2 are satisfied. 
3.2. Gradient estimates. In [30], a local gradient estimate is given by using the local geometry of $M$. Inspired from this, local gradient estimates of $P_{s, t} f$ are studied by using local version of derivative formula, and the corresponding result is presented as follows.

Theorem 3.4. Let $\mathcal{R}_{s}^{Z} \geq K_{s}$ for some $K \in C\left(\left[0, T_{c}\right) \times M\right)$. Given $x \in M$, let

$$
\kappa_{s}(x)=\sup _{r \in[s, s+1]}\left(\sup _{B_{r}(x, 1)} K(r, \cdot)^{-}+\left|Z_{r}\right|_{r}(x)\right) .
$$

Then there exists a constant $c>0$ such that for any $f \in \mathcal{B}_{b}(M)$,

$$
\left|\nabla^{s} P_{s, t} f\right|_{s}(x) \leq \frac{\|f\|_{\infty} \exp \left[c\left(1+\kappa_{s}(x)\right)\right]}{\sqrt{(t-s) \wedge 1}} .
$$

Proof. Without loss of generality, we consider $s=0$ for simplicity. By the semigroup property and the contraction of $P_{s, t}$, it suffices to prove (3.12) for $0<t \leq 1 \wedge T_{c}$. It is easy to see that if we choose some explicit process $h$ in Theorem 3.1, then the associated gradient estimate of $P_{s, t} f$ can be achieved by only using local geometry of the manifold. To this end, for $x \in M$, let $D=\{(r, y) \in[0, t] \times M$ : $\left.\rho_{r}(x, y) \leq 1\right\}$. It is easy to see that $D$ is closed and hence compact, since $\rho_{r}(x, y)$ is continuous in $(r, x, y)$ (see [23, Lemma 2.5]). Let $\varphi\left(t, X_{t}\right)=\cos \left(\pi \rho_{t}\left(x, X_{t}\right) / 2\right)$. Let $X_{0}=x$ and

$$
T(r)=\left(\int_{0}^{r} \varphi^{-2}\left(t, X_{t}\right) d t\right) \mathbf{1}_{\left\{r \leq \tau_{D}\right\}}+\infty \mathbf{1}_{\left\{r>\tau_{D}\right\}}
$$

where $\tau_{D}$ is the first hitting time of $\left(r, X_{r}\right)$ to $\partial D$. Define

$$
\tau(r)=\inf \{u \geq 0: T(u) \geq r\}, \quad r \geq 0 .
$$

Then $T \circ \tau(r)=r$ for $r \leq \tau_{D}$. Moreover,

$$
\tau^{\prime}(r)=\frac{1}{T^{\prime} \circ \tau(r)}=\varphi^{2}\left(\tau(r), X_{\tau(r)}\right), \quad r \leq \tau_{D}
$$

Since $\varphi \leq 1$, we have $\tau(r) \leq r$. Define $h(r)=\frac{1}{t} \int_{0}^{r \wedge \tau(t)} \varphi^{-2}\left(u, X_{u}\right) d u$. Then, $h$ meets the requirement of Theorem 3.1 and

$$
\int_{0}^{\tau(t)} h^{\prime}(r)^{2} d r=\frac{1}{t^{2}} \int_{0}^{\tau(t)} \varphi^{-4}\left(r, X_{r}\right) d r=\frac{1}{t^{2}} \int_{0}^{\tau(t)} \varphi^{-2}\left(r, X_{r}\right) d T(r)=\frac{1}{t^{2}} \int_{0}^{t} \varphi^{-2}\left(\tau(r), X_{\tau(r)}\right) d r .
$$

Moreover, let $v \in T_{x} M$ and $|v|_{0}=1$. By the definition of $Q_{r}$ and $\mathcal{R}_{r}^{Z} \geq-\kappa_{0}$ on $D$, we have

$$
\left|u_{r} Q_{r} u_{0}^{-1} v\right|_{r} \leq|v|_{0} e^{\kappa_{0}}, \quad r \leq \tau(t), \quad 0<t \leq 1 .
$$

Combining this with Theorem 3.1, we have

$$
\begin{aligned}
\left|\left\langle\nabla^{0} P_{0, t} f(x), v\right\rangle_{0}\right| & \leq \frac{1}{\sqrt{2}}\|f\|_{\infty} e^{\kappa_{0}(x)}\left(\mathbb{E}^{x} \int_{0}^{\tau(t)} h^{\prime}(r)^{2} d r\right)^{1 / 2} \\
& =\frac{1}{\sqrt{2}}\|f\|_{\infty} e^{\kappa_{0}(x)} \frac{1}{t^{2}} \int_{0}^{t} \varphi^{-2}\left(\tau(r), X_{\tau(r)}\right) d r .
\end{aligned}
$$

Thus, it suffices for us to estimate the last term above. Before this, we prove that $\left(\tau(r), X_{\tau(r)}\right)$ is non-explosive on $D$, i.e. the life time $\tau:=\inf \left\{r \geq 0,\left(\tau(r), X_{\tau(r)}\right) \in \partial D\right\}$ satisfies $\tau=\infty$, a.e.

For $n \geq 1$, let $\tau_{n}=\inf \left\{r: \varphi\left(\tau(r), X_{\tau(r)}\right) \leq 1 / n\right\}$. Note that $X_{\tau(r)}$ is generated by $\varphi^{2} L_{\tau(r)}$, then by (3.11), there exists some constant $c>0$ such that

$$
\varphi^{2}\left(L_{\tau(r)}+\partial_{1}\right) \varphi^{-1}=-\left(L_{\tau(r)}+\partial_{1}\right) \varphi+2 \varphi^{-1}\left|\nabla^{\tau(r)} \varphi\right|_{\tau(r)}^{2} \leq c \varphi^{-1},
$$

where $\partial_{1}$ denotes the derivative with respect to the first variable. Hence, we have

$$
\mathbb{E}^{x} \varphi^{-1}\left(\tau\left(t \wedge \tau_{n}\right), X_{\tau\left(t \wedge \tau_{n}\right)}\right) \leq \varphi^{-1}(0, x) e^{c t}=e^{c t}, \quad t \geq 0, n \geq 1 .
$$


On the other hand, $\mathbb{E}^{x} \varphi^{-1}\left(\tau\left(t \wedge \tau_{n}\right), X_{\tau\left(t \wedge \tau_{n}\right)}\right) \geq n \mathbb{P}\left(\tau_{n}<t\right)$. By this and (3.14), it is easy to see that

$$
\mathbb{P}^{x}\left(\tau_{n}<t\right) \leq n^{-1} e^{c t} \text {. }
$$

Letting $n \rightarrow \infty$, we have $\mathbb{P}^{x}(\tau<t)=0, t \geq 0$, i.e. $\mathbb{P}^{x}(\tau=\infty)=1$.

Next, by using the Itô formula,

$$
d \varphi^{-2}\left(\tau(r), X_{\tau(r)}\right) \leq d M_{r}+\left[\varphi^{2}\left(L_{\tau(r)}+\partial_{1}\right) \varphi^{-2}\right]\left(\tau(r), X_{\tau(r)}\right) d r
$$

holds for some local martingale $M_{r}$. According to (3.11) and the definition of $\kappa$, there exists a constant $c_{1}>0$ such that for all $r \in[0, t]$,

$$
\sin \left(\pi \rho_{r}(x, \cdot) / 2\right)\left(L_{r}+\partial_{r}\right) \rho_{r}(x, \cdot) \leq c_{1}\left(1+\kappa_{0}(x)\right)
$$

holds on $D$. Thus, there exists a constant $c_{2}>0$ such that for all $r \in[0, t]$,

$$
\varphi^{2}\left(L_{r}+\partial_{r}\right) \varphi^{-2}=-2 \varphi^{-1}\left(L_{r}+\partial_{r}\right) \varphi+6 \varphi^{-2}\left|\nabla^{r} \varphi\right|_{r}^{2} \leq c_{2}\left(1+\kappa_{0}(x)\right) \varphi^{-2}
$$

holds on $D$. Combining this with (3.13) and (3.15), we obtain that for $t \in(0,1]$,

$$
\begin{aligned}
\mathbb{E}^{x} \int_{0}^{\tau(t)} h^{\prime}(r)^{2} d r & =\frac{1}{t^{2}} \int_{0}^{t} \mathbb{E}^{x} \varphi^{-2}\left(\tau(r), X_{\tau(r)}\right) d r \\
& \leq \frac{1}{t^{2}} \int_{0}^{t} e^{c_{2}\left(1+\kappa_{0}(x)\right) r} d r \leq \frac{c_{3}}{t} e^{c_{3}\left(1+\kappa_{0}(x)\right)}
\end{aligned}
$$

for some constant $c_{3}>0$. From this, it follows that

$$
\left|\left\langle\nabla^{0} P_{0, t} f(x), v\right\rangle_{0}\right| \leq\|f\|_{\infty} e^{\kappa_{0}(x)}\left(\mathbb{E} \int_{0}^{\tau(t)} h^{\prime}(r)^{2} d r\right)^{1 / 2} \leq \frac{\|f\|_{\infty} c_{4} e^{c_{4}\left(1+\kappa_{0}(x)\right)}}{\sqrt{t}}
$$

holds for some constant $c_{4}>0$ and all $t \in(0,1]$. This completes the proof.

Remark 3.5. We would like to indicate that recently, the author with X. Chen and J. Mao give local gradient estimates for some important geometric quantities under Ricci flow, mean curvature flow and Yamabe flow by using some probabilistic method, see [11] for more details.

Next, we aim to provide various equivalent semigroup inequalities for the lower bound curvature condition, i.e.

$$
\mathcal{R}_{t}^{Z} \geq K_{t}, \text { for some } K \in C\left(\left[0, T_{c}\right) \times M\right) .
$$

By using the derivative formula, we have

Theorem 3.6. Assume $(A 1)$ or $(A 2)$ holds. Let $p \geq 1$ and $\tilde{p}=p \wedge 2$. Then for any $K \in C\left(\left[0, T_{c}\right) \times M\right)$ and $t \in\left[0, T_{c}\right)$ such that $K_{t}(x)^{-} / \rho_{t}^{2}(x) \rightarrow 0$ as $\rho_{t}(x) \rightarrow \infty$, the following statements are equivalent to each other.

(i) The curvature condition (3.17) holds for the function $K$.

(ii) For any $x \in M, 0 \leq s \leq t<T_{c}$ and $f \in C_{c}^{1}(M)$,

$$
\left|\nabla^{s} P_{s, t} f(x)\right|_{s}^{p} \leq \mathbb{E}^{(s, x)}\left\{\left|\nabla^{t} f\right|_{t}^{p}\left(X_{t}\right) e^{-p \int_{s}^{t} K\left(r, X_{r}\right) d r}\right\} .
$$

(iii) For any $0 \leq s \leq t<T_{c}, x \in M$ and positive function $f \in C_{c}^{1}(M)$,

$$
\frac{\tilde{p}\left[P_{s, t} f^{2}-\left(P_{s, t} f^{2 / \tilde{p}}\right)^{\tilde{p}}\right]}{4(\tilde{p}-1)} \leq \mathbb{E}^{(s, x)}\left\{\left|\nabla^{t} f\right|_{t}^{2}\left(X_{t}\right) \int_{s}^{t} e^{-2 \int_{u}^{t} K\left(r, X_{r}\right) d r} d u\right\},
$$

where when $p=1$, the inequality is understood as its limit as $p \downarrow 1$ :

$$
P_{s, t}\left(f^{2} \log f^{2}\right)(x)-\left(P_{s, t} f^{2} \log P_{s, t} f^{2}\right)(x) \leq 4 \mathbb{E}^{(s, x)}\left\{\left|\nabla^{t} f\right|_{t}^{2}\left(X_{t}\right) \int_{s}^{t} e^{-2 \int_{u}^{t} K\left(r, X_{r}\right) d r} d u\right\} .
$$


(iv) For any $0 \leq s \leq t<T_{c}, x \in M$ and positive function $f \in C_{c}^{1}(M)$,

$$
\left|\nabla^{s} P_{s, t} f\right|_{s}^{2}(x) \leq \frac{\left[P_{s, t} f^{\tilde{p}}-\left(P_{s, t} f\right)^{\tilde{p}}\right](x)}{\tilde{p}(\tilde{p}-1) \int_{s}^{t}\left(\mathbb{E}^{(s, x)}\left\{\left(P_{u, t} f\right)^{2-\tilde{p}}\left(X_{u}\right) e^{-2 \int_{s}^{u} K\left(r, X_{r}\right) d r}\right\}\right)^{-1} d u},
$$

where when $p=1$, the inequality is understood as its limit as $p \downarrow 1$ :

$$
\left|\nabla^{s} P_{s, t} f\right|_{s}^{2}(x) \leq \frac{\left[P_{s, t}(f \log f)-\left(P_{s, t} f\right) \log P_{s, t} f\right](x)}{\int_{s}^{t}\left(\mathbb{E}^{(s, x)}\left\{P_{u, t} f\left(X_{u}\right) e^{-2 \int_{s}^{u} K\left(r, X_{r}\right) d r}\right\}\right)^{-1} d u} .
$$

Remark 3.7. Consider a special case: $L=\Delta$ and $K=0$, i.e., $g_{t}$ is a super Ricci flow. Then Theorem 3.6(ii)-(iv) give equivalent characterizations for the super Ricci flow. It is worth mentioning that very recently R. Haslhofer and A. Naber [20] use a sharp infinite dimensional gradient estimate to characterize solutions of the Ricci flow.

To prove this result, we first need to characterize $\mathcal{R}_{t}^{Z}$ by using the Taylor expansions first. When the metric is independent of $t$, the following results are essentially due to [3, 32].

Lemma 3.8. For $s \in\left[0, T_{c}\right)$ and $x \in M$, let $X \in T_{x} M$ with $|X|_{s}=1$. Let $f \in C_{0}^{\infty}(M)$ such that $\nabla^{s} f(x)=X$ and $\operatorname{Hess}_{f}^{s}(x)=0$, and let $f_{n}=n+f$ for $n \geq 1$. Then,

(i) for any $p>0$,

$$
\mathcal{R}_{s}^{Z}(X, X)=\lim _{t \downarrow s} \frac{P_{s, t}\left|\nabla^{t} f\right|_{t}^{p}(x)-\left|\nabla^{s} P_{s, t} f\right|_{s}^{p}(x)}{p(t-s)}
$$

(ii) for any $p>1$,

$$
\begin{aligned}
\mathcal{R}_{s}^{Z}(X, X) & =\lim _{n \rightarrow \infty} \lim _{t \downarrow s} \frac{1}{t-s}\left(\frac{p\left\{P_{s, t} f_{n}^{2}-\left(P_{s, t} f_{n}^{\frac{2}{p}}\right)^{p}\right\}}{4(p-1)(t-s)}-\left|\nabla^{s} P_{s, t} f_{n}\right|_{s}^{2}\right)(x) \\
& =\lim _{n \rightarrow \infty} \lim _{t \downarrow s} \frac{1}{t-s}\left(P_{s, t}\left|\nabla^{t} f\right|_{t}^{2}-\frac{p\left\{P_{s, t} f_{n}^{2}-\left(P_{s, t} f_{n}^{\frac{2}{p}}\right)^{p}\right\}}{4(p-1)(t-s)}\right)(x) ;
\end{aligned}
$$

(iii) $\mathcal{R}_{s}^{Z}(X, X)$ is equal to each of the following limits:

$$
\begin{aligned}
& \lim _{n \rightarrow \infty} \lim _{t \downarrow s} \frac{1}{(t-s)^{2}}\left\{\left(P_{s, t} f_{n}\right)\left[P_{s, t}\left(f_{n} \log f_{n}\right)-\left(P_{s, t} f_{n}\right) \log P_{s, t} f_{n}\right]-(t-s)\left|\nabla^{s} P_{s, t} f\right|_{s}^{2}\right\}(x) ; \\
& \lim _{n \rightarrow \infty} \lim _{t \downarrow s} \frac{1}{4(t-s)^{2}}\left\{4(t-s) P_{s, t}\left|\nabla^{t} f\right|_{t}^{2}+\left(P_{s, t} f_{n}^{2}\right) \log P_{s, t} f_{n}^{2}-P_{s, t} f_{n}^{2} \log f_{n}^{2}\right\}(x) .
\end{aligned}
$$

Proof. (a). Without loss of generality, we only prove for $s=0$. Since $\nabla^{0} f=X$ and $\operatorname{Hess}_{f}^{0}(x)=0$, by the Bochner-Weitzenböck formula, we have

$$
\Gamma_{2}^{0}(f, f)(x):=\frac{1}{2} L_{0}\left|\nabla^{0} f\right|_{0}^{2}(x)-\left\langle\nabla^{0} f, \nabla^{0} L_{0} f\right\rangle_{0}(x)=\operatorname{Ric}_{0}(X, X)-\left\langle\nabla_{X}^{0} Z_{0}, X\right\rangle_{0} .
$$

Thus, the first assertion follows from the Taylor expansions at point $x$ (we drop $x$ below for simplicity):

$$
P_{0, t}\left|\nabla^{t} f\right|_{t}^{p}=\left|\nabla^{0} f\right|_{0}^{p}+\left(\frac{p}{2}\left|\nabla^{0} f\right|_{0}^{p-2} L_{0}\left|\nabla^{0} f\right|_{0}^{2}-\left.\frac{p}{2}\left|\nabla^{0} f\right|_{0}^{p-2} \partial_{t} g_{t}\right|_{t=0}\left(\nabla^{0} f, \nabla^{0} f\right)\right) t+\mathrm{o}(t),
$$

and

$$
\left|\nabla^{0} P_{0, t} f\right|_{0}^{p}=\left|\nabla^{0} f\right|_{0}^{p}+p t\left|\nabla^{0} f\right|_{0}^{p-2}\left\langle\nabla^{0} L_{0} f, \nabla^{0} f\right\rangle_{0}+\mathrm{o}(t),
$$

where in the first equality we use the following equality,

$$
\partial_{t}\left|\nabla^{t} f\right|_{t}^{2}=-\partial_{t} g_{t}\left(\nabla^{t} f, \nabla^{t} f\right)
$$


(b). Let $f_{n}=n+f$, which is positive for large $n$. Then, for small $t>0$ and large $n$,

$$
\begin{aligned}
P_{0, t} f_{n}^{2}-\left(P_{0, t} f_{n}^{2 / p}\right)^{p}= & \frac{8(p-1) t^{2}}{p}\left\langle\nabla^{0} f, \nabla^{0} L_{0} f\right\rangle_{0}-\left.\frac{2(p-1) t^{2}}{p} \partial_{t} g_{t}\right|_{t=0}\left(\nabla^{0} f, \nabla^{0} f\right) \\
& +\frac{4(p-1) t}{p}\left|\nabla^{0} f\right|_{0}^{2}+\frac{4(p-1) t^{2}}{p} \Gamma_{2}^{0}(f, f)+t^{2} \mathrm{O}\left(n^{-1}\right)+\mathrm{o}\left(t^{2}\right) .
\end{aligned}
$$

By this and (3.21) for $p=2$, we prove the first equality in (3.19). Similarly, the second equality follows from (3.20) for $p=2$.

(c). The equalities in (iii) can be proved by combining (3.20) and (3.21) for $p=2$ with the following two asymptotic formulae respectively.

$$
\begin{aligned}
& \left(P_{0, t} f_{n}\right)\left\{P_{0, t}\left(f_{n} \log f_{n}\right)-\left(P_{0, t} f_{n}\right) \log P_{0, t} f_{n}\right\} \\
= & t\left|\nabla^{0} f\right|_{0}^{2}+t^{2} \Gamma_{2}^{0}(f, f)+2 t^{2}\left\langle\nabla^{0} f, \nabla^{0} L_{0} f\right\rangle_{0}-\left.\frac{1}{2} t^{2} \partial_{t} g_{t}\right|_{t=0}\left(\nabla^{0} f, \nabla^{0} f\right)+t^{2} \mathrm{O}\left(n^{-2}\right)+\mathrm{o}\left(t^{2}\right) ; \\
& \left(P_{0, t} f_{n}^{2}\right) \log P_{0, t} f_{n}^{2}-P_{0, t}\left(f_{n}^{2} \log f_{n}^{2}\right) \\
= & -4 t\left|\nabla^{0} f\right|_{0}^{2}-4 t^{2}\left\langle\nabla^{0} L_{0} f, \nabla^{0} f\right\rangle_{0}+\left.2 t^{2} \partial_{t} g_{t}\right|_{t=0}\left(\nabla^{0} f, \nabla^{0} f\right)-2 t^{2} L_{0}\left|\nabla^{0} f\right|_{0}^{2}+\mathrm{o}\left(t^{2}\right)+t^{2} \mathrm{O}\left(n^{-1}\right) .
\end{aligned}
$$

Proof of Theorem 3.6 According to the proof of Theorem 3.2, $\mathbb{E}^{(s, x)} \exp \left(p \int_{s}^{t} K^{-}\left(s, X_{s}\right) d s\right)<\infty$ holds for any $p>0,0 \leq s \leq t<T_{c}$ and $x \in M$. So, we obtain (i) of Lemma 3.8 by applying (ii) to $f \in C_{0}^{\infty}(M)$ such that $\operatorname{Hess}_{f}^{s}(x)=0$ or applying (iii) to $n+f$ in place of $f$, or applying (iv) to $(f+n)^{2 / p}$ when $p>1$ (resp. $f+n$ when $p=1$ ) in place of $f$. Thus, it suffices to show that (i) implies (ii)-(iv).

First, if $\mathcal{R}_{t}^{Z} \geq K_{t}, t \in\left[0, T_{c}\right.$ ), then by the first equality in (3.9) and (3.1), we have

$$
\begin{aligned}
\left|\nabla^{s} P_{s, t} f\right|_{s}(x) & \leq \mathbb{E}^{(s, x)}\left\{\left|\nabla^{t} f\right|_{t}\left(X_{t}\right) \exp \left[-\int_{s}^{t} K\left(u, X_{u}\right) d u\right]\right\} \\
& \leq \mathbb{E}^{(s, x)}\left\{\left|\nabla^{t} f\right|_{t}^{p}\left(X_{t}\right) \exp \left[-p \int_{s}^{t} K\left(u, X_{u}\right) d u\right]\right\}^{1 / p},
\end{aligned}
$$

thus, (ii) holds.

To prove (iii) and (iv), let $p \in(1,2]$. Without loss of generality, we only prove for $s=0$. By Theorem 2.1 and (ii) with $p=1$, for $0<u<\zeta_{n}\left(\zeta_{n}\right.$ is defined as in (2.2) with $s=0$ ),

$$
\begin{aligned}
d\left(P_{u, t} f^{2 / p}\right)^{p}\left(X_{u}\right) & \\
& =d M_{u}+\left(L_{u}+\partial_{u}\right)\left(P_{u, t} f^{2 / p}\right)^{p}\left(X_{u}\right) d u \\
& =d M_{u}+p(p-1)\left(P_{u, t} f^{2 / p}\right)^{p-2}\left(X_{u}\right)\left|\nabla^{u} P_{u, t} f^{2 / p}\right|_{u}^{2}\left(X_{u}\right) d u \\
& \leq d M_{u}+p(p-1)\left(P_{u, t} f^{2 / p}\right)^{p-2}\left(X_{u}\right) \mathbb{E}^{\left(u, X_{u}\right)}\left\{\frac{2}{p} f^{\frac{2-p}{p}}\left(X_{t}\right)\left|\nabla^{t} f\right|_{t}\left(X_{t}\right) \mathrm{e}^{-\int_{u}^{t} K\left(r, X_{r}\right) d r}\right\}^{2} d u \\
& \leq d M_{u}+\frac{4(p-1)}{p}\left(P_{u, t} f^{2 / p}\right)^{p-2}\left(P_{u, t} f^{2(2-p) / p}\right)\left(X_{u}\right) \mathbb{E}^{\left(u, X_{u}\right)}\left(\left|\nabla^{t} f\right|_{t}^{2}\left(X_{t}\right) e^{-2 \int_{u}^{t} K\left(r, X_{r}\right) d r}\right) d u,
\end{aligned}
$$

where $M_{u}$ is the local martingale part of $\left(P_{u, t} f^{2 / p}\right)^{p}\left(X_{u}\right)$. Moreover, since $2-p \in[0,1]$, by the Jensen inequality,

$$
P_{u, t} f^{2(2-p) / p} \leq\left(P_{u, t} f^{2 / p}\right)^{2-p},
$$

we then arrive at

$$
d\left(P_{u, t} f^{2 / p}\right)^{p}\left(X_{u}\right) \leq d M_{u}+\frac{4(p-1)}{p} \mathbb{E}^{\left(u, X_{u}\right)}\left(\left|\nabla^{t} f\right|_{t}^{2}\left(X_{t}\right) e^{-2 \int_{u}^{t} K\left(r, X_{r}\right) d r}\right) d u, \quad u<\zeta_{n} .
$$


By taking integral over $\left[0, s \wedge \zeta_{n}\right]$, this implies

$$
\mathbb{E}^{x}\left(P_{s \wedge \zeta_{n}, t} f^{2 / p}\right)^{p}\left(X_{s \wedge \zeta_{n}}\right)-\left(P_{0, t} f^{2 / p}\right)^{p}(x) \leq \int_{0}^{s \wedge \zeta_{n}} \frac{4(p-1)}{p} \mathbb{E}^{x}\left[\left|\nabla^{t} f\right|_{t}^{2}\left(X_{t}\right) \exp \left(-2 \int_{u}^{t} K\left(r, X_{r}\right) d r\right)\right] d u \text {. }
$$

Letting $n \rightarrow \infty$, we obtain

$$
\frac{d}{d u} P_{0, u}\left(P_{u, t} f^{2 / p}\right)^{p}(x) \leq \frac{4(p-1)}{p} \mathbb{E}^{x}\left\{\left|\nabla^{t} f\right|_{t}^{2}\left(X_{t}\right) e^{-2 \int_{u}^{t} K\left(r, X_{r}\right) d r}\right\}, \quad u \in[0, t] .
$$

This implies (iii) for $s=0$ by taking integral over $[0, t]$.

Similarly,

$$
d\left(P_{u, t} f\left(X_{u}\right)\right)^{p}=d \tilde{M}_{u}+p(p-1)\left(P_{u, t} f\right)^{p-1}\left(X_{u}\right)\left|\nabla^{u} P_{u, t} f\right|_{u}^{2}\left(X_{u}\right) d u, \quad 0<u<\zeta_{n},
$$

where $\tilde{M}_{u}$ is the local martingale part of $\left(P_{u, t} f\left(X_{u}\right)\right)^{p}$, which, together with (ii), implies

$$
\frac{d}{d u} P_{0, u}\left(P_{u, t} f\right)^{p}=p(p-1) P_{0, u}\left\{\left(P_{u, t} f\right)^{p-2}\left|\nabla^{u} P_{u, t} f\right|_{u}^{2}\right\} .
$$

Thus,

$$
\begin{aligned}
\frac{d}{d u} P_{0, u}\left(P_{u, t} f\right)^{p} & \geq \frac{p(p-1)\left\{\mathbb{E}^{x}\left[\left|\nabla^{u} P_{u, t} f\right|_{u}\left(X_{u}\right) e^{-\int_{0}^{u} K\left(r, X_{r}\right) d r}\right]\right\}^{2}}{\mathbb{E}^{x}\left\{\left(P_{u, t} f\right)^{2-p}\left(X_{u}\right) e^{-2 \int_{0}^{u} K\left(r, X_{r}\right) d r}\right\}} \\
& \geq \frac{p(p-1)\left|\nabla^{0} P_{0, t} f\right|_{0}^{2}}{\mathbb{E}^{x}\left\{\left(P_{u, t} f\right)^{2-p}\left(X_{u}\right) e^{-2 \int_{0}^{u} K\left(r, X_{r}\right) d r}\right\}} .
\end{aligned}
$$

Integrating over $[0, t]$, we prove (iv) for $s=0$.

3.3. Dimension-free Harnack inequalities. The study of dimension-free Harnack inequalities was initiated in [33], which is applied to characterize some important properties of the underlying processes, see [38]. Note that when the metric is fixed, the following equivalence of (i) and (iv) are essentially due to [34]. In what follows, we simply write $p_{s, t}(x, y)=p(s, x ; t, y)$.

Theorem 3.9. Let $p \in(1, \infty)$ and $K \in C\left(\left[0, T_{c}\right)\right)$. Then the following assertions are equivalent to each other.

(i) The curvature condition (3.17) holds for the function $K$.

(ii) For any $f \in \mathcal{B}_{b}^{+}(M)$ and $0 \leq s \leq t<T_{c}$,

$$
\left(P_{s, t} f\right)^{p}(x) \leq P_{s, t} f^{p}(y) \exp \left\{\frac{p}{4(p-1)}\left[\int_{s}^{t} e^{2 \int_{s}^{r} K(u) d u} d r\right]^{-1} \rho_{s}^{2}(x, y)\right\} .
$$

(iii) For any $f \in \mathcal{B}_{b}^{+}(M)$ with $f \geq 1$ and $0 \leq s \leq t<T_{c}$,

$$
P_{s, t} \log f(x) \leq \log P_{s, t} f(y)+\left[4 \int_{s}^{t} e^{2 \int_{s}^{r} K(u) d u} d r\right]^{-1} \rho_{s}^{2}(x, y) .
$$

(iv) For any $0 \leq s \leq t<T_{c}$ and $x, y \in M$,

$$
\int_{M} p_{s, t}(x, y)\left(\frac{p_{s, t}(x, y)}{p_{s, t}(y, z)}\right)^{\frac{1}{p-1}} \mu_{t}(d z) \leq \exp \left\{\frac{p}{4(p-1)^{2}}\left[\int_{s}^{t} e^{2 \int_{s}^{r} K(u) d u} d r\right]^{-1} \rho_{s}^{2}(x, y)\right\} .
$$

(v) For any $0 \leq s \leq t<T_{c}$ and $x, y \in M$,

$$
\int_{M} p_{s, t}(x, y) \log \frac{p_{s, t}(x, y)}{p_{s, t}(y, z)} \mu_{t}(d z) \leq\left[4 \int_{s}^{t} e^{2 \int_{s}^{r} K(u) d u} d r\right]^{-1} \rho_{s}^{2}(x, y) .
$$


Proof. By [37, Proposition 2.4], (ii) and (iii) are equivalent to (iv) and (v) respectively. Moreover, according to [38, Corollary 1.4.3], we see that (ii) implies (iii). Thus, it is sufficient to prove that "(i) $\Rightarrow$ (ii)" and "(iii) $\Rightarrow$ (i)".

(a) (i) implies (ii). We consider the case for $s=0$. By approximation and the monotone class theorem, we may assume that $f \in C^{2}(M)$, inf $f>0$ and $f$ is constant outside a compact set. Given $x \neq y$ and $t>0$, let $\gamma:[0, t] \rightarrow M$ be the constant speed $g_{0}$-geodesic from $x$ to $y$ with length $\rho_{0}(x, y)$. Let $v_{s}=\frac{d \gamma_{s}}{d s}$. Then we have $\left|v_{s}\right|_{0}=\rho_{0}(x, y) / t$. Let

$$
h(s)=\frac{t \int_{0}^{s} e^{2 \int_{0}^{r} K(u) d u} d r}{\int_{0}^{t} e^{2 \int_{0}^{r} K(u) d u} d r} .
$$

Then $h(0)=0$ and $h(t)=t$. Let $y_{s}=\gamma_{h(s)}$ and

$$
\varphi(s)=\log P_{0, s}\left(P_{s, t} f\right)^{p}\left(y_{s}\right), s \in[0, t] .
$$

To get $\varphi^{\prime}(s)$, we first see that

$$
\begin{aligned}
d\left(P_{s, t} f\left(X_{s}\right)\right)^{p} & =d M_{s}+\left(L_{s}+\partial_{s}\right)\left(P_{s, t} f\right)^{p}\left(X_{s}\right) d s \\
& =d M_{s}+p(p-1)\left(P_{s, t} f\right)^{p-2}\left(X_{s}\right)\left|\nabla^{s} P_{s, t} f\right|_{s}^{2}\left(X_{s}\right) d s, \quad s<\zeta_{n},
\end{aligned}
$$

where $M_{s}$ is the local martingale part of $\left(P_{s, t} f\left(X_{s}\right)\right)^{p}$, which implies that

$$
\mathbb{E}^{x}\left(P_{s \wedge \zeta_{n}, t} f\left(X_{s \wedge \zeta_{n}}\right)\right)^{p}-\left(P_{0, t} f\right)^{p}(x)=p(p-1) \mathbb{E}^{x} \int_{0}^{s \wedge \zeta_{n}}\left(P_{u, t} f\right)^{p-2}\left(X_{u}\right)\left|\nabla^{u} P_{u, t} f\right|_{u}^{2}\left(X_{u}\right) d u .
$$

Moreover, due to Theorem 3.6 (ii), it holds $\left|\nabla^{u} P_{u, t} f\right|_{u} \leq e^{-\int_{u}^{t} K(r) d r} P_{u, t}\left|\nabla^{t} f\right|_{t}$. From this and inf $f>0$, we deduce by letting $n \rightarrow \infty$ that

$$
\mathbb{E}^{x}\left(P_{s, t} f\left(X_{s}\right)\right)^{p}-\left(P_{0, t} f\right)^{p}(x)=p(p-1) \int_{0}^{s} \mathbb{E}^{x}\left[\left(P_{u, t} f\right)^{p-2}\left(X_{u}\right)\left|\nabla^{u} P_{u, t} f\right|_{u}^{2}\left(X_{u}\right)\right] d u .
$$

By this and the Kolmogorov equations, we obtain

$$
\begin{aligned}
\frac{d \varphi(s)}{d s} & =\frac{1}{P_{0, s}\left(P_{s, t} f\right)^{p}}\left\{p(p-1) P_{0, s}\left(P_{s, t} f\right)^{p}\left|\nabla^{s} \log P_{s, t} f\right|_{s}^{2}+h^{\prime}(s)\left\langle\nabla^{0} P_{0, s}\left(P_{s, t} f\right)^{p}, v_{s}\right\rangle_{0}\right\} \\
& \geq \frac{p}{P_{0, s}\left(P_{s, t} f\right)^{p}} P_{0, s}\left\{\left(P_{s, t} f\right)^{p}\left((p-1)\left|\nabla^{s} \log P_{s, t} f\right|_{s}^{2}-\frac{\rho_{0}(x, y)}{t} h^{\prime}(s) e^{-\int_{0}^{s} K(u) d u}\left|\nabla^{s} \log P_{s, t} f\right|_{s}\right)\right\} \\
& \geq \frac{-p \rho_{0}(x, y)^{2} h^{\prime}(s)^{2} e^{-2 \int_{0}^{s} K(u) d u}}{4(p-1) t^{2}},
\end{aligned}
$$

for $s \in[0, t]$. Since $h^{\prime}(s)=\frac{t e^{2} \int_{0}^{s} K(u) d u}{\int_{0}^{t} e^{2} \int_{0}^{r} K(u) d u} d r$, we arrive at

$$
\frac{d \varphi(s)}{d s} \geq \frac{-p \rho_{0}(x, y)^{2} e^{\int_{0}^{s} 2 K(u) d u}}{4(p-1)\left(\int_{0}^{t} e^{2 \int_{0}^{r} K(u) d u} d r\right)^{2}}, \quad s \in[0, t] .
$$

By integrating over $s$ from 0 and $t$, we complete the proof.

(b) (iii) implies (i). Suppose that $s=0$. Let $x \in M$ and $X \in T_{x} M$ be fixed. For any $n \geq 1$, we may take $f \in C^{\infty}(M)$ such that $f \geq n, f$ is constant outside a compact set, and

$$
\nabla^{0} f(x)=X, \operatorname{Hess}_{f}^{0}(x)=0 .
$$

Taking $\gamma_{t}=\exp _{x}\left[-2 t \nabla^{0} \log f(x)\right]$, we have $\rho_{0}\left(x, \gamma_{t}\right)=2 t\left|\nabla^{0} \log f\right|_{0}(x)$ for $t \in\left[0, t_{0}\right]$, where $t_{0}$ is a positive constant such that $\rho_{0}\left(x, \gamma_{t}\right)<r$ and $r>0$. By using (iv) with $y=\gamma_{t}$, we obtain

$$
P_{0, t}(\log f)(x) \leq \log P_{0, t} f\left(\gamma_{t}\right)+\frac{t^{2}\left|\nabla^{0} \log f\right|_{0}^{2}(x)}{\int_{0}^{t} e^{2 \int_{0}^{r} K(u) d u} d r}, \quad t \in\left[0, t_{0}\right] .
$$


Since $L_{0} f \in C_{0}^{2}(M)$ and $\operatorname{Hess}_{f}^{0}(x)=0$ implies $\nabla^{0}\left|\nabla^{0} f\right|_{0}^{2}(x)=0$ at point $x$, by Taylor's expansion, we have

$$
P_{0, t}(\log f)(x)=\log f(x)+t\left(f^{-1} L_{0} f-\left|\nabla^{0} \log f\right|_{0}^{2}\right)(x)+\frac{t^{2}}{2} A+\mathrm{o}\left(t^{2}\right)
$$

for small $t>0$, where

$$
\begin{aligned}
A:= & \frac{L_{0}^{2} f}{f}-\frac{\left(L_{0} f\right)^{2}}{f^{2}}-\frac{2}{f^{2}}\left\langle\nabla^{0} L_{0} f, \nabla^{0} f\right\rangle_{0}-\frac{L_{0}\left|\nabla^{0} f\right|_{0}^{2}}{f^{2}}+\frac{4\left|\nabla^{0} f\right|_{0}^{2} L_{0} f}{f^{3}} \\
& -\frac{6\left|\nabla^{0} f\right|_{0}^{4}}{f^{4}}+\frac{\left.\partial_{t} g_{t}\right|_{t=0}\left(\nabla^{0} f, \nabla^{0} f\right)}{f^{2}}+\left.\frac{1}{f} \frac{d L_{t} f}{d t}\right|_{t=0} .
\end{aligned}
$$

On the other hand, let $N_{t}=P_{x, \gamma_{t}}^{0} \nabla^{0} \log f(x)$, where $P_{x, \gamma_{t}}^{0}$ is the $g_{0}$-parallel displacement along the $g_{0}$-geodesic $\gamma: t \rightarrow \gamma_{t}$. We have $\dot{\gamma}_{t}=-2 N_{t}$ and $\nabla_{\dot{\gamma}_{t}}^{0} N_{t}=0$. Thus, note that $\operatorname{Hess}_{f}^{0}(x)=0$, the Taylor expansion of $\log P_{0, t} f\left(\gamma_{t}\right)$ at $x$ is the following

$$
\log P_{0, t} f\left(\gamma_{t}\right)=\log f(x)+t\left(f^{-1} L_{0} f-2\left|\nabla^{0} \log f\right|_{0}^{2}\right)(x)+\frac{t^{2}}{2} B+\mathrm{o}\left(t^{2}\right),
$$

where

$$
B:=\frac{L_{0}^{2} f}{f}-\frac{\left(L_{0} f\right)^{2}}{f^{2}}+\frac{4 L_{0} f\left|\nabla^{0} f\right|_{0}^{2}}{f^{3}}-\frac{4\left\langle\nabla^{0} L_{0} f, \nabla^{0} f\right\rangle_{0}}{f^{2}}-4 \frac{\left|\nabla^{0} f\right|_{0}^{4}}{f^{4}}+\left.\frac{1}{f} \frac{d L_{t} f}{d t}\right|_{t=0} .
$$

Combining this with (3.23) and (3.24), we arrive at

$$
\begin{aligned}
& \frac{1}{t}\left(1-\frac{t}{\int_{0}^{t} e^{2 \int_{0}^{r} K(u) d u} d r}\right)\left|\nabla^{0} \log f\right|_{0}^{2}(x) \\
& \leq \frac{1}{2}\left(\frac{L_{0}\left|\nabla^{0} f\right|_{0}^{2}-2\left\langle\nabla^{0} L_{0} f, \nabla^{0} f\right\rangle_{0}}{f^{2}}+\frac{2\left|\nabla^{0} f\right|_{0}^{4}}{f^{4}}+\left.\frac{1}{f^{2}} \partial_{t} g_{t}\right|_{t=0}\left(\nabla^{0} f, \nabla^{0} f\right)\right)(x)+\mathrm{o}(1) .
\end{aligned}
$$

Letting $t \rightarrow 0$, we obtain

$$
\frac{1}{2} L_{0}\left|\nabla^{0} f\right|_{0}^{2}(x)-\left\langle\nabla^{0} L_{0} f, \nabla f\right\rangle_{0}(x) \geq K(0)\left|\nabla^{0} f\right|_{0}^{2}(x)+\left.\frac{1}{2} \partial_{t} g_{t}\right|_{t=0}\left(\nabla^{0} f, \nabla^{0} f\right)(x)-\frac{\left|\nabla^{0} f\right|_{0}^{4}}{f^{2}}(x) .
$$

By the Bochner-Weitzenböck formula, it follows that for $n \geq 1$,

$$
\operatorname{Ric}_{0}(X, X)-\left\langle\nabla_{X}^{0} Z_{0}, X\right\rangle_{0} \geq K(0)|X|_{0}^{2}+\left.\frac{1}{2} \partial_{t} g_{t}\right|_{t=0}(X, X)-\frac{|X|_{0}^{4}}{n} .
$$

This implies (i) for $s=0$ by letting $n \rightarrow \infty$.

Remark 3.10. Let $p_{x, y}^{s, t}(z)=\frac{p_{s, t}(x, z)}{p_{s, t}(y, z)}$ for $x, y, z \in M$ and $0 \leq s \leq t<T_{c}$. According to [37, Proposition 2.4], we have the following statements, which are equivalent to Theorem 3.9 (ii)(iii) respectively.

(ii') For any $0 \leq s \leq t<T_{c}, p_{x, y}^{s, t}$ satisfies

$$
P_{s, t}\left(\left(p_{x, y}^{s, t}\right)^{1 /(\alpha-1)}\right)(x) \leq\left\{\frac{p}{p-1}\left[4 \int_{s}^{t} e^{2 \int_{s}^{r} K(u) d u} d r\right]^{-1} \rho_{s}^{2}(x, y)\right\}^{1 /(\alpha-1)}, x, y \in M .
$$

(iii') For any $0 \leq s \leq t<T_{c}, p_{x, y}^{s, t}$ satisfies

$$
P_{s, t}\left\{\log p_{x, y}^{s, t}\right\}(x) \leq\left[4 \int_{s}^{t} e^{2 \int_{s}^{r} K(u) d u} d r\right]^{-1} \rho_{s}^{2}(x, y), \quad x, y \in M .
$$


3.4. Other functional inequalities. In [6] functional inequalities of the following type are shown to be useful on manifolds with a fixed metric. We now extend this type of results to our case.

Theorem 3.11. Let $K \in C\left(\left[0, T_{c}\right)\right)$. The following assertions are equivalent to each other.

(i) The curvature condition (3.17) holds for the function $K$.

(ii) For any $0 \leq s \leq r \leq t<T_{c}$ and $1<q_{1} \leq q_{2}$ such that

$$
\frac{q_{2}-1}{q_{1}-1}=\frac{\int_{s}^{t} e^{2 \int_{s}^{u} K(\tau) d \tau} d u}{\int_{s}^{r} e^{2 \int_{s}^{u} K(\tau) d \tau} d u}
$$

it holds

$$
\left\{P_{s, r}\left(P_{r, t} f\right)^{q_{2}}\right\}^{\frac{1}{q_{2}}} \leq\left(P_{s, t} f^{q_{1}}\right)^{\frac{1}{q_{1}}}
$$

for all positive function $f \in \mathcal{B}_{b}(M)$.

(iii) For any $0 \leq s \leq r \leq t<T_{c}$ and $0<q_{2} \leq q_{1}$ or $q_{2} \leq q_{1}<0$ such that (3.25) is satisfied,

$$
\left(P_{s, t} f^{q_{1}}\right)^{\frac{1}{q_{1}}} \leq\left\{P_{s, r}\left(P_{r, t} f\right)^{q_{2}}\right\}^{\frac{1}{q_{2}}}
$$

for all positive function $f \in \mathcal{B}_{b}(M)$.

Proof. From Theorem 3.6, we know that (i) is equivalent to Theorem 3.6 (iii) with $p=1$ for $K \in$ $C\left(\left[0, T_{c}\right)\right)$. Thus, it suffices for us to show that each of (ii) and (iii) is equivalent to Theorem 3.6 (iii) with $p=1$ for $K \in C\left(\left[0, T_{c}\right)\right)$.

(a) Theorem 3.6 (iii) with $p=1$ for $K \in C\left(\left[0, T_{c}\right)\right.$ ) implies (ii) and (iii). We again prove this assertion for $s=0$. By an approximation argument, it suffices to prove for $f \in C^{\infty}(M)$ such that inf $f>0$ and $f$ is constant outside a compact set. In this case, given $t \in\left(0, T_{c}\right)$, let

$$
q(s)=1+\frac{\left(q_{1}-1\right) \int_{0}^{t} e^{2 \int_{0}^{r} K(u) d u} d r}{\int_{0}^{s} e^{2 \int_{0}^{r} K(u) d u} d r} \text { and } \psi(s)=\left\{P_{0, s}\left(P_{s, t} f\right)^{q(s)}\right\}^{\frac{1}{q(s)}},
$$

for all $s \in(0, t]$. Then

$$
\int_{0}^{s} e^{-2 \int_{r}^{s} K(u) d u} d r+\frac{q(s)-1}{q^{\prime}(s)}=0,
$$

which together with (3.22) implies

$$
\begin{aligned}
\left(\frac{\psi^{\prime} \psi^{q-1} q^{2}}{q^{\prime}}\right)(s)= & P_{0, s}\left(P_{s, t} f\right)^{q(s)} \log \left(P_{s, t} f\right)^{q(s)}-P_{0, s}\left(P_{s, t} f\right)^{q(s)} \log P_{0, s}\left(P_{s, t} f\right)^{q(s)} \\
& +\frac{q(s)^{2}(q(s)-1)}{q^{\prime}(s)} P_{0, s}\left(P_{s, t} f\right)^{q(s)-2}\left|\nabla^{s} P_{s, t} f\right|_{s}^{2} .
\end{aligned}
$$

Due to Theorem 3.6 (iii) with $p=1$ for $K \in C\left(\left[0, T_{c}\right)\right)$, we further have

$$
\left(\frac{\psi^{\prime} \psi^{q-1} q^{2}}{q^{\prime}}\right)(s) \leq q(s)^{2}\left(\int_{0}^{s} e^{-2 \int_{u}^{t} K(r) d r} d u+\frac{q(s)-1}{q^{\prime}(s)}\right) P_{0, s}\left(P_{s, t} f\right)^{q(s)-2}\left|\nabla^{s} P_{s, t} f\right|_{s}^{2}=0 .
$$

Therefore, in case (ii) one has $q^{\prime}(s)<0$ so that $\psi^{\prime}(s) \geq 0$, while in case (iii) one has $q^{\prime}(s)>0$ so that $\psi^{\prime}(s) \leq 0$. Hence, the inequalities in (ii) and (iii) hold.

(b) (ii) or (iii) implies Theorem 3.6 (iii) with $p=1$ for $K \in C\left(\left[0, T_{c}\right)\right.$ ). We only prove that (ii) implies (iii), since "(ii) implies (iii)" can be shown in a similar way. We also only consider $s=0$. Let $q_{1}=2$ and $q_{2}=2(1+\varepsilon)$ for small $\varepsilon>0$. According to (3.25), we take $r(\varepsilon)$ such that

$$
\frac{1}{1+2 \varepsilon}=\frac{\int_{0}^{r(\varepsilon)} e^{2 \int_{0}^{u} K(\tau) d \tau} d u}{\int_{0}^{t} e^{2 \int_{0}^{u} K(\tau) d \tau} d u}=1-\frac{\int_{r(\varepsilon)}^{t} e^{2 \int_{0}^{u} K(\tau) d \tau} d u}{\int_{0}^{t} e^{2 \int_{0}^{u} K(\tau) d \tau} d u} .
$$


Then,

$$
\frac{2 \varepsilon}{1+2 \varepsilon} \int_{0}^{t} e^{2 \int_{0}^{u} K(\tau) d \tau} d u=\int_{r(\varepsilon)}^{t} e^{2 \int_{0}^{u} K(\tau) d \tau} d u \sim(t-r(\varepsilon)) e^{2 \int_{0}^{t} K(\tau) d \tau}
$$

i.e.

$$
(t-r(\varepsilon)) \sim 2 \varepsilon \int_{0}^{t} e^{-2 \int_{u}^{t} K(\tau) d \tau} d u
$$

So, we obtain from (ii) that

$$
\begin{aligned}
0 & \geq \lim _{\varepsilon \rightarrow 0} \frac{1}{\varepsilon}\left\{\left[P_{0, r(\varepsilon)}\left(P_{r(\varepsilon), t}\right)^{2(1+\varepsilon)}\right]^{\frac{1}{(1+\varepsilon)}}-\left(P_{0, t} f^{2}\right)\right\} \\
& =P_{0, t} f^{2} \log f^{2}-\left(P_{0, t} f^{2}\right) \log P_{0, t} f^{2}-4 \int_{0}^{t} e^{-2 \int_{u}^{t} K(r) d r} d u \cdot P_{0, t}\left|\nabla^{t} f\right|_{t}^{2} .
\end{aligned}
$$

Therefore, Theorem 3.6 (iii) with $p=1$ holds for $K \in C\left(\left[0, T_{c}\right)\right)$.

\section{COUPLING FOR $L_{t}$-DIFFUSION PROCESSES AND ITS APPLICATIONS}

4.1. Coupling processes. We aim to construct coupling processes for $L_{t}$-diffusion processes by parallel translation and mirror reflection in this subsection.

Let us introduce some basic notions first. Recall that $\mathrm{Cut}_{t}(x)$ is the set of the $g_{t}$-cut-locus of $x$ on $M$. Then, for each $t \in\left[0, T_{c}\right)$, the $g_{t}$-cut-locus $\mathrm{Cut}_{t}$ and the space time cut-locus Cut ${ }_{\mathrm{ST}}$ are defined by

$$
\begin{aligned}
\mathrm{Cut}_{t} & =\left\{(x, y) \in M \times M \mid y \in \mathrm{Cut}_{t}(x)\right\} ; \\
\mathrm{Cut}_{\mathrm{ST}} & =\left\{(t, x, y) \in\left[0, T_{c}\right) \times M \times M \mid(x, y) \in \mathrm{Cut}_{t}\right\} .
\end{aligned}
$$

Set $D(M)=\{(x, x) \mid x \in M\}$. For any $(x, y) \notin$ Cut $_{t}$ with $x \neq y$, let $\left\{J_{i}^{t}\right\}_{i=1}^{d-1}$ be Jacobi fields along the minimal geodesic $\gamma$ from $x$ to $y$ with respect to the metric $g_{t}$, such that $\left\{J_{i}^{t}, \dot{\gamma}: 1 \leq i \leq d-1\right\}$ is an orthonormal basis at $x$ and $y$. Let

$$
\begin{aligned}
I_{Z}(t, x, y)= & \sum_{i=1}^{d-1} \int_{\gamma}\left(\left\langle\nabla_{\dot{\gamma}}^{t} J_{i}^{t}, \nabla_{\dot{\gamma}}^{t} J_{i}^{t}\right\rangle_{t}-\left\langle R_{t}\left(J_{i}^{t}, \dot{\gamma}\right) \dot{\gamma}, J_{i}^{t}\right\rangle_{t}+\frac{1}{2} \partial_{t} g_{t}(\dot{\gamma}, \dot{\gamma})\right)(\gamma(s)) d s \\
& +Z_{t} \rho_{t}(y, \cdot)(x)+Z_{t} \rho_{t}(x, \cdot)(y),
\end{aligned}
$$

where $R_{t}$ is the curvature tensor with respect to the metric $g_{t}$. Moreover, let $P_{x, y}^{t}: T_{x} M \rightarrow T_{y} M$ be the $g_{t}$-parallel translation along the geodesic $\gamma$. Define the $g_{t}$-mirror reflection by

$$
M_{x, y}^{t}: T_{x} M \rightarrow T_{y} M ; v \mapsto P_{x, y}^{t} v-2\langle v, \dot{\gamma}\rangle_{t}(x) \dot{\gamma}(y) .
$$

It is well known that $P_{x, y}^{t}$ and $M_{x, y}^{t}$ are smooth outside $\mathrm{Cut}_{t}$ and $D(M)$. For convenience, we set $P_{x, x}^{t}$ and $M_{x, x}^{t}$ be the identity for $x \in M$. Our main result in this subsection is the following.

Theorem 4.1. Let $x \neq y$ and $0<T<T_{c}$ be fixed. Let $U:[0, T] \times M \times M \rightarrow T M$ be $C^{1}$-smooth in $\left(\text { Cut }_{\mathrm{ST}} \cup[0, T] \times D(M)\right)^{c}$ such that $U\left(t, x_{1}, x_{2}\right) \in T_{x_{2}} M$ for $\left(t, x_{1}, x_{2}\right) \in[0, T] \times M \times M$.

(i) There exist two $\mathbb{R}^{d}$-valued Brownian motions $B_{t}$ and $\tilde{B}_{t}$ on a complete filtered probability space $\left(\Omega,\left\{\mathscr{F}_{t}\right\}_{t \geq 0}, \mathbb{P}\right)$ such that

$$
\mathbf{1}_{\left\{\left(X_{t}, \tilde{X}_{t}\right) \notin \mathrm{Cut}_{t}\right\}} d \tilde{B}_{t}=\mathbf{1}_{\left\{\left(X_{t}, \tilde{X}_{t}\right) \notin \mathrm{Cut}_{t}\right\}} \tilde{u}_{t}^{-1} P_{X_{t}, \tilde{X}_{t}}^{t} u_{t} d B_{t},
$$

where $X_{t}$ with lift $u_{t}$ and $\tilde{X}_{t}$ with lift $\tilde{u}_{t}$ solve the following equation

$$
\begin{cases}d X_{t}=\sqrt{2} u_{t} \circ d B_{t}+Z_{t}\left(X_{t}\right) d t, & X_{0}=x, \\ d \tilde{X}_{t}=\sqrt{2} \tilde{u}_{t} \circ d \tilde{B}_{t}+\left\{Z_{t}\left(\tilde{X}_{t}\right)+U\left(t, X_{t}, \tilde{X}_{t}\right) \mathbf{1}_{\left\{X_{t} \neq \tilde{X}_{t}\right\}}\right\} d t, & \tilde{X}_{0}=y .\end{cases}
$$


Moreover, for any $J \in C([0, T] \times M \times M)$ such that $J \geq I_{Z}$ on $\left(\mathrm{Cut}_{\mathrm{ST}} \cup[0, T] \times D(M)\right)^{c}$,

$$
d \rho_{t}\left(X_{t}, \tilde{X}_{t}\right) \leq\left\{J\left(t, X_{t}, \tilde{X}_{t}\right)+\left\langle U\left(t, X_{t}, \tilde{X}_{t}\right), \nabla^{t} \rho_{t}\left(X_{t}, \cdot\right)\left(\tilde{X}_{t}\right)\right\rangle_{t} \mathbf{1}_{\left\{X_{t} \neq \tilde{X}_{t}\right\}}\right\} d t
$$

holds up to the coupling time $T_{0}:=\inf \left\{t \in[0, T]: X_{t}=\tilde{X}_{t}\right\}$ with convention $\inf \varnothing=T$.

(ii) The first assertion in (1) holds by replacing $P_{X_{t}, \tilde{X}_{t}}^{t}$ with $M_{X_{t}, \tilde{X}_{t}}^{t}$. In this case, for any $J \in$ $C([0, T] \times M \times M)$ such that $J \geq I_{Z}$ on $\left(\mathrm{Cut}_{\mathrm{ST}} \cup[0, T] \times D(M)\right)^{c}$,

$$
d \rho_{t}\left(X_{t}, \tilde{X}_{t}\right) \leq 2 \sqrt{2} d b_{t}+\left\{J\left(t, X_{t}, \tilde{X}_{t}\right)+\left\langle U\left(t, X_{t}, \tilde{X}_{t}\right), \nabla^{t} \rho_{t}\left(X_{t}, \cdot\right)\left(\tilde{X}_{t}\right)\right\rangle_{t} \mathbf{1}_{\left\{X_{t} \neq \tilde{X}_{t}\right\}}\right\} d t
$$

holds up to the coupling time $T_{0}$, where $b_{t}$ is a one-dimensional Brownian motion.

Proof. We first deal with the reflecting coupling case for $U=0$. For the parallel coupling case, the proof is similar by replacing $M_{x, y}^{t}$ with $P_{x, y}^{t}$ below. The proof is divided into two parts.

Part I: Construction of $\left(X_{t}, \tilde{X}_{t}\right)$. Let $u_{t}$ and $X_{t}:=\mathbf{p} u_{t}$ solve (2.1). To get rid of the trouble that $M_{x, y}^{t}$ does not exist on $\mathrm{Cut}_{t} \cup D(M)$, we modify this operator so that it vanishes in a neighborhood of these sets. To this end, for any $n \geq 1$ and $\varepsilon \in(0,1)$, let $h_{n, \varepsilon} \in C^{\infty}([0, T] \times M \times M)$ so that

$$
0 \leq h_{n, \varepsilon} \leq(1-\varepsilon),\left.h_{n, \varepsilon}\right|_{C_{n}^{c}}=1-\varepsilon, \text { and }\left.h_{n, \varepsilon}\right|_{C_{2 n}}=0 \text {, }
$$

where

$$
C_{n}:=\left\{(t, x, y) \in[0, T] \times M \times M: \mathbf{d}\left((t, x, y), \mathrm{Cut}_{\mathrm{ST}}\right) \leq 1 / n\right\}
$$

and $\mathbf{d}$ is a distance on $[0, T] \times M \times M$ such that, for $\left(s, x_{1}, y_{1}\right),\left(t, x_{2}, y_{2}\right) \in[0, T] \times M \times M$,

$$
\mathbf{d}\left(\left(s, x_{1}, y_{1}\right),\left(t, x_{2}, y_{2}\right)\right)=|t-s|+\sup _{r \in[0, T]} \rho_{r}\left(x_{1}, x_{2}\right)+\sup _{r \in[0, T]} \rho_{r}\left(y_{1}, y_{2}\right) .
$$

Moreover, let $\varphi_{n} \in C^{\infty}([0, T] \times M \times M)$ such that $0 \leq \varphi_{n} \leq 1,\left.\varphi_{n}\right|_{D_{2 n}}=0$ and $\left.\varphi_{n}\right|_{D_{n}^{c}}=1$, where

$$
D_{n}:=\left\{(t, x, y) \in[0, T] \times M \times M: \rho_{t}(x, y) \leq \frac{1}{n}\right\} .
$$

Let $\tilde{u}_{t}^{n, \varepsilon}$ and $\tilde{X}_{t}^{n, \varepsilon}:=\mathbf{p} \tilde{u}_{t}^{n, \varepsilon}$ solve the SDE

$$
\left\{\begin{aligned}
& d \tilde{u}_{t}^{n, \varepsilon}= \sqrt{2}\left(h_{n, \varepsilon} \varphi_{n}\right)\left(t, X_{t}, \tilde{X}_{t}^{n, \varepsilon}\right) \sum_{i=1}^{d} H_{i}^{t}\left(\tilde{u}_{t}^{n, \varepsilon}\right) \circ d \tilde{B}_{t}^{i}-\frac{1}{2} \sum_{\alpha, \beta} \mathcal{G}_{\alpha, \beta}\left(t, \tilde{u}_{t}^{n, \varepsilon}\right) V_{\alpha \beta}\left(\tilde{u}_{t}^{n, \varepsilon}\right) d t \\
&+\sqrt{2\left[1-\left(h_{n, \varepsilon} \varphi_{n}\right)^{2}\left(t, X_{t}, \tilde{X}_{t}^{n, \varepsilon}\right)\right]} \sum_{i=1}^{d} H_{i}^{t}\left(\tilde{u}_{t}^{n, \varepsilon}\right) \circ d B_{t}^{\prime i}+H_{Z_{t}}^{t}\left(\tilde{u}_{t}^{n, \varepsilon}\right) d t, \\
& \tilde{u}_{0}^{n, \varepsilon} \in O_{t}(M), \mathbf{p} \tilde{u}_{0}^{n, \varepsilon}=y,
\end{aligned}\right.
$$

where $B_{t}^{\prime}$ is a Brownian motion on $\mathbb{R}^{d}$ independent of $B_{t}$, and on $\left(\mathrm{Cut}_{\mathrm{ST}}\right)^{c}$,

$$
d \tilde{B}_{t}=\left(\tilde{u}_{t}^{n, \varepsilon}\right)^{-1} M_{X_{t}, \tilde{X}_{t}^{n, \varepsilon}}^{t} u_{t} d B_{t} .
$$

Since the coefficients involved in (4.2) are at least $C^{1}$, there exists a solution $\tilde{u}_{t}^{n, \varepsilon}$ solving the equation (4.2). Let

$$
\tilde{L}_{n, \varepsilon}(t):=\Delta_{t}(x)+\Delta_{t}(y)+Z_{t}(x)+Z_{t}(y)+h_{n, \varepsilon} \varphi_{n}(t, x, y) \sum_{i, j=1}^{d}\left\langle M_{x, y}^{t} V_{i}, W_{j}\right\rangle_{t} V_{i} W_{j},
$$

where $\left\{V_{i}\right\}$ and $\left\{W_{i}\right\}$ are orthonormal bases at $x$ and $y$ respectively. It is easy to see that $\left(X_{t}, \tilde{X}_{t}^{n, \varepsilon}\right)$ is generated by $\tilde{L}_{n, \varepsilon}(t)$ and hence, is a coupling of $L_{t}$-diffusion processes as the marginal operators of $\tilde{L}_{n, \varepsilon}(t)$ coincide with $L_{t}$.

Now, let $\mathbb{P}_{n, \varepsilon}^{x, y}$ be the distribution of $\left(X_{t}, \tilde{X}_{t}^{n, \varepsilon}\right)$, which is a probability measure on the path space $M_{x}^{T} \times M_{y}^{T}$, where $M_{x}^{T}:=\left\{\gamma \in C([0, T], M): \gamma_{0}=x\right\}$ is equipped with the $\sigma$-field $\mathscr{F}_{x}^{T}$ induced by all measurable cylindric functions. Since $\left\{\mathbb{P}_{n, \varepsilon}^{x, y}: n \geq 1,0<\varepsilon<1\right\}$ is a family of couplings for $\mathbb{P}^{x}$ and $\mathbb{P}^{y}$, 
it is easy to see that $\left\{\mathbb{P}_{n, \varepsilon}^{x, y}: n \geq 1,0<\varepsilon<1\right\}$ is tight. Then, for each $\varepsilon>0$, there exists a probability measure $\mathbb{P}_{\varepsilon}^{x, y}$ and a subsequence $\left\{n_{k}\right\}$ such that $\mathbb{P}_{n_{k}, \varepsilon}^{x, y}$ converges weakly to $\mathbb{P}_{\varepsilon}^{x, y}$ as $k \rightarrow \infty$. Meanwhile, there exists a subsequence $\left\{\varepsilon_{l}\right\}$ such that $\mathbb{P}_{\varepsilon_{l}}^{x, y}$ converges weakly to some $\mathbb{P}^{x, y}$ as $l \rightarrow \infty$. Let

$$
\tilde{L}_{t}(x, y)=L_{t}(x)+L_{t}(y)+1_{\left(\mathrm{Cut}_{t} \cup D(M)\right)^{c}}(x, y) \sum_{i, j=1}^{d}\left\langle M_{x, y}^{t} V_{i}, W_{j}\right\rangle_{t} V_{i} W_{j} .
$$

Then it is easy to see that $\mathbb{P}^{x, y}$ solves the martingale problem for $\tilde{L}_{t}$ up to the coupling time, i.e. for any $f \in C_{0}^{\infty}(M \times M \backslash D(M))$,

$$
f\left(\xi_{t}, \eta_{t}\right)-\int_{0}^{t} \tilde{L}_{s} f\left(\xi_{s}, \eta_{s}\right) d s, \quad 0 \leq t \leq T
$$

is a $\mathbb{P}^{x, y}$-martingale with respect to the natural filtration up to the coupling time. Here and in the sequel, $(\xi ., \eta$.) $\in C([0, T] ; M \times M)$ is the canonical path. It is well known that solutions to martingale problem for $\tilde{L}_{s}$ can be constructed as solutions to a stochastic differential equation. Therefore, there exist two independent $d$-dimensional Brownian motions $B_{t}$ and $B_{t}^{\prime}$ on a complete probability space $\left(\Omega, \mathscr{F}, \mathscr{F}_{t}, \mathbb{P}\right)$, and two processes $X_{t}, \tilde{X}_{t}$ such that

$$
\begin{cases}d^{\mathrm{It} \hat{0}} X_{t}=\sqrt{2} u_{t} d B_{t}+Z_{t}\left(X_{t}\right) d t, & X_{0}=x, \\ d^{\mathrm{It} \hat{0}} \tilde{X}_{t}=\sqrt{2} \mathbf{1}_{\mathrm{Cut}_{t}^{c}}\left(X_{t}, \tilde{X}_{t}\right) M_{X_{t}, \tilde{X}_{t}}^{t} u_{t} d B_{t}+\sqrt{2} \mathbf{1}_{\mathrm{Cut}_{t}}\left(X_{t}, \tilde{X}_{t}\right) \tilde{u}_{t} d B_{t}^{\prime}+Z_{t}\left(\tilde{X}_{t}\right) d t, & \tilde{X}_{0}=y\end{cases}
$$

holds up to $T_{0}:=\inf \left\{t \in[0, T]: X_{t}=\tilde{X}_{t}\right\}$, where $d^{\text {Itô }}$ stands for the Itô differential operator. By letting $\tilde{X}_{t}=X_{t}$ after $T_{0}$, we complete the proof of the first assertion.

Part II: Proof of (4.1). (a) We only consider noncompact $M$. For the compact case, the proof is simpler by dropping the stopping time $\tau$ below. Since the generator $\tilde{L}_{n, \varepsilon}(t)$ is strictly elliptic and all the coefficients involved are at least $C^{1}$, and hence by Remark 2.2, $\mathbb{P}^{x, y}\left(\left(X_{t}, \tilde{X}_{t}^{n, \varepsilon}\right) \in \cdot\right)$ has a density $p_{t}^{n, \varepsilon}(z, w)$ with respect to $\mu_{t} \otimes \mu_{t}$. Moreover, Cut $t_{t}$ is closed and $\mu_{t} \otimes \mu_{t}\left(\mathrm{Cut}_{t}\right)=0$, then it is easy to see that the set

$$
\left\{t \in[0, T] \mid\left(t, X_{t}, \tilde{X}_{t}^{n, \varepsilon}\right) \in \mathrm{Cut}_{\mathrm{ST}}\right\}
$$

has Lebesgue measure zero almost surely. This implies $\mathbf{1}_{\mathrm{Cut} t}\left(X_{t}, \tilde{X}_{t}^{n, \varepsilon}\right)=0$, a.s. Then, by [25, Theorem 2] (the Itô formula for radial process $\left.\rho_{t}\left(x, X_{t}\right), x \in M\right)$, we have

$$
\begin{aligned}
d \rho_{t}\left(X_{t}, \tilde{X}_{t}^{n, \varepsilon}\right)= & \sqrt{2} \sum_{i=1}^{d} h_{n, \varepsilon} \varphi_{n}\left(M_{X_{t}, \tilde{X}_{t}^{n, \varepsilon}}^{t} u_{t} e_{i}\right) \rho_{t}\left(X_{t}, \cdot\right)\left(\tilde{X}_{t}^{n, \varepsilon}\right) d B_{t}^{i}+\sqrt{2} \sum_{i=1}^{d} u_{t} e_{i} \rho_{t}\left(\cdot, \tilde{X}_{t}^{n, \varepsilon}\right)\left(X_{t}\right) d B_{t}^{i} \\
& +\sqrt{2\left[1-\left(h_{n, \varepsilon} \varphi\right)^{2}\right]\left(t, X_{t}, \tilde{X}_{t}^{n, \varepsilon}\right)} \sum_{i=1}^{d} \tilde{u}_{t}^{n, \varepsilon} e_{i} \rho_{t}\left(X_{t}, \cdot\right)\left(\tilde{X}_{t}^{n, \varepsilon}\right) d B_{t}^{\prime i} \\
& +\left(\tilde{L}_{n, \varepsilon}(t)+\partial_{t}\right) \rho_{t}\left(X_{t}, \tilde{X}_{t}^{n, \varepsilon}\right) d t-d l_{t}^{n, \varepsilon} .
\end{aligned}
$$

This implies

$$
\begin{aligned}
d \rho_{t}\left(X_{t}, \tilde{X}_{t}^{n, \varepsilon}\right) \leq & \sqrt{2\left(h_{n, \varepsilon} \varphi_{n}+1\right)^{2}+2\left[1-\left(h_{n, \varepsilon} \varphi_{n}\right)^{2}\right]} d b_{t}^{n, \varepsilon}-d l_{t}^{n, \varepsilon} \\
& +\left[h_{n, \varepsilon} \varphi_{n} I_{Z}+\left(1-h_{n, \varepsilon} \varphi_{n}\right) S\right]\left(t, X_{t}, \tilde{X}_{t}^{n, \varepsilon}\right) d t \\
= & 2 \sqrt{\left(h_{n, \varepsilon} \varphi_{n}\right)\left(t, X_{t}, \tilde{X}_{t}^{n, \varepsilon}\right)+1} d b_{t}^{n, \varepsilon}-d l_{t}^{n, \varepsilon} \\
& +\left[h_{n, \varepsilon} \varphi_{n} I_{Z}+\left(1-h_{n, \varepsilon} \varphi_{n}\right) S\right]\left(t, X_{t}, \tilde{X}_{t}^{n, \varepsilon}\right) d t,
\end{aligned}
$$

where $b_{t}^{n, \varepsilon}$ is a one-dimensional Brownian motion, $l_{t}^{n, \varepsilon}$ is an increasing process which increases only when $\left(X_{t}, \tilde{X}_{t}^{n, \varepsilon}\right) \in \mathrm{Cut}_{t}$, and for $(t, x, y) \notin \mathrm{Cut}_{\mathrm{ST}}$,

$$
S(t, x, y):=L_{t} \rho_{t}(\cdot, y)(x)+L_{t} \rho_{t}(x, \cdot)(y)+\partial_{t} \rho_{t}(x, y) .
$$


Let $\mathbf{B}$ be a fixed bounded smooth open domain in $M$. Given $N \geq 1$, the Laplacian comparison theorem implies that there exists a constant $C>0$ such that $S(t, x, y) \leq C$ for all $(t, x, y) \in([0, T] \times \overline{\mathbf{B}} \times \overline{\mathbf{B}}) \cap$ $\left(\mathrm{Cut}_{\mathrm{ST}} \cup D_{N}\right)^{c}$. Then, it follows from (4.5) that, when $\left(t, X_{t}, \tilde{X}_{t}^{n, \varepsilon}\right) \in([0, T] \times \mathbf{B} \times \mathbf{B}) \cap D_{N}^{c}$ and $n \geq N$,

$$
\begin{aligned}
d \rho_{t}\left(X_{t}, \tilde{X}_{t}^{n, \varepsilon}\right)= & 2 \sqrt{\left(h_{n, \varepsilon} \varphi_{n}\right)\left(t, X_{t}, \tilde{X}_{t}^{n, \varepsilon}\right)+1} d b_{t}^{n, \varepsilon}-d \tilde{l}_{t}^{n, \varepsilon} \\
& +\left[h_{n, \varepsilon} \varphi_{n} J+\left(1-h_{n, \varepsilon} \varphi_{n}\right) C\right]\left(t, X_{t}, \tilde{X}_{t}^{n, \varepsilon}\right) d t
\end{aligned}
$$

where $\tilde{l}_{t}^{n, \varepsilon}$ is a larger increasing process. Now let $f \in C^{2}(\mathbb{R})$ with $f^{\prime} \geq 0$ and $\left.f^{\prime}\right|_{[0,1 / N]}=0$. By the Itô formula, we obtain from (4.6) that, for the coordinate process $\left(\xi_{t}, \eta_{t}\right)$ with $\tau:=\inf \left\{t \geq 0:\left(\xi_{t}, \eta_{t}\right) \notin\right.$ $\mathbf{B} \times \mathbf{B}\}$ and any $n \geq N$,

$$
\begin{aligned}
S_{t}^{n, \varepsilon}(f):= & f \circ \rho_{t}\left(\xi_{t \wedge \tau}, \eta_{t \wedge \tau}\right) \\
& -\int_{0}^{t \wedge \tau}\left\{2\left(h_{n, \varepsilon}+1\right) f^{\prime \prime} \circ \rho+\left[h_{n, \varepsilon} J+\left(1-h_{n, \varepsilon}\right) C\right] f^{\prime} \circ \rho\right\}\left(s, \xi_{s}, \eta_{s}\right) d s
\end{aligned}
$$

is a $\mathbb{P}_{n, \varepsilon}^{x, y}$-supermartingale, where $\rho(t, \cdot, \cdot):=\rho_{t}(\cdot, \cdot), t \in[0, T]$. Thus, for any $0 \leq t^{\prime}<t \leq T$ and $\mathscr{F}_{t^{\prime}}$-measurable nonnegative $\phi \in C_{b}\left(M_{x}^{T} \times M_{y}^{T}\right)$, one has

$$
\mathbb{E}_{n, \varepsilon}^{x, y} \phi S_{t}^{n, \varepsilon}(f) \leq \mathbb{E}_{n, \varepsilon}^{x, y} \phi S_{t^{\prime}}^{n, \varepsilon}(f), n \geq N,
$$

where $\mathbb{E}_{n, \varepsilon}^{x, y}$ is the expectation with respect to $\mathbb{P}_{n, \varepsilon}^{x, y}$.

(b) Since the strict ellipticity of $\tilde{L}_{n, \varepsilon}(t)$ is uniform in $n$, and all coefficients of this operator are uniformly bounded in $n$ on any compact set $K \subset M \times M$, the density $p_{t}^{n, \varepsilon}, t \in(0, T]$ satisfies a Harnack inequality uniform in $n$ on $K$ (see [31, Theorem 1] ). That is for each $t \in(0, T]$, there exists a constant $C>0$ such that $p_{t}^{n, \varepsilon} \leq C$ on $K$ for all $n \geq 1$. Let $G$ be an open set containing Cut $_{t}$. Then,

$$
\mathbb{P}_{\varepsilon}^{x, y}\left(\left(\xi_{t}, \eta_{t}\right) \in G \cap K^{\circ}\right)=\varliminf_{k \rightarrow \infty} \mathbb{P}_{n_{k}, \varepsilon}^{x, y}\left(\left(\xi_{t}, \eta_{t}\right) \in G \cap K^{\circ}\right) \leq C \mu_{t} \otimes \mu_{t}\left(G \cap K^{\circ}\right),
$$

where $K^{\circ}$ is the inner set of $K$. Since $\mathrm{Cut}_{t}$ is a closed set of measure zero with respect to $\mu_{t} \otimes \mu_{t}$, by letting $G \rightarrow \mathrm{Cut}_{t}$ and then $K \rightarrow M \times M$, we obtain

$$
\mathbb{P}_{\varepsilon}^{x, y}\left(\left(t, \xi_{t}, \eta_{t}\right) \in \mathrm{Cut}_{\mathrm{ST}}\right)=\mathbb{P}_{\varepsilon}^{x, y}\left(\left(\xi_{t}, \eta_{t}\right) \in \mathrm{Cut}_{t}\right)=0 .
$$

Therefore, we have, for any $\delta>0$, there exists $m \geq 1$ such that

$$
\int_{0}^{t} \mathbb{P}_{\varepsilon}^{x, y}\left(\left(s, \xi_{s}, \eta_{s}\right) \in C_{m}\right) d s \leq \delta .
$$

Moreover, it is easy to see that $C_{m}$ is closed since $\mathbf{d}$ is continuous and $\mathrm{Cut}_{\mathrm{ST}}$ is closed (see [28]), which implies

$$
\varlimsup_{k \rightarrow \infty} \mathbb{P}_{n_{k}, \varepsilon}^{x, y}\left(\left(s, \xi_{s}, \eta_{s}\right) \in C_{m}\right) \leq \mathbb{P}_{\varepsilon}^{x, y}\left(\left(s, \xi_{s}, \eta_{s}\right) \in C_{m}\right), \quad 0 \leq s \leq T .
$$

With this and (4.8), we obtain that

$$
\varlimsup_{k \rightarrow \infty} \int_{0}^{t} \mathbb{P}_{n_{k}, \varepsilon}^{x, y}\left(\left(s, \xi_{s}, \eta_{s}\right) \in C_{m}\right) d s \leq \delta .
$$

Let

$$
S_{t}^{\varepsilon}(f)=f \circ \rho_{t}\left(\xi_{t \wedge \tau}, \eta_{t \wedge \tau}\right)-\int_{0}^{t} \mathbf{1}_{\{s<\tau\}}\left[2(2-\varepsilon) f^{\prime \prime} \circ \rho+((1-\varepsilon) J+\varepsilon C) f^{\prime} \circ \rho\right]\left(s, \xi_{s}, \eta_{s}\right) d s .
$$

\footnotetext{
${ }^{1}$ By a localization argument, there exist finite local coordinates covering the compact set $K$. Meanwhile, the corresponding heat kernel is uniformly bounded in $n$ on any local coordinates by [31, Theorem 1] and the reference measures are equivalent to each other on $K$, thus the heat kernel is uniformly bounded in $n$ on the compact set $K$.
} 
Combining this with (4.7), (4.8) and (4.9), and noting that $h_{n, \varepsilon}=1-\varepsilon$ on $C_{m}^{c}$ for $n \geq m$, we obtain that there exists some constant $c_{1}>0$, such that

$$
\begin{aligned}
& \mathbb{E}_{\varepsilon}^{x, y} S_{t}^{\varepsilon}(f) \phi \\
= & \mathbb{E}_{\varepsilon}^{x, y} \phi\left\{f \circ \rho_{t}\left(\xi_{t \wedge \tau}, \eta_{t \wedge \tau}\right)-\int_{0}^{t} \mathbf{1}_{\{s<\tau\}}\left[2(2-\varepsilon) f^{\prime \prime} \circ \rho+((1-\varepsilon) J+\varepsilon C) f^{\prime} \circ \rho\right]\left(s, \xi_{s}, \eta_{s}\right) d s\right\} \\
= & \lim _{k \rightarrow \infty} \mathbb{E}_{n_{k}, \varepsilon}^{x, y} \phi\left\{f \circ \rho_{t}\left(\xi_{t \wedge \tau}, \eta_{t \wedge \tau}\right)-\int_{0}^{t} \mathbf{1}_{\{s<\tau\}}\left[2(2-\varepsilon) f^{\prime \prime} \circ \rho+((1-\varepsilon) J+\varepsilon C) f^{\prime} \circ \rho\right]\left(s, \xi_{s}, \eta_{s}\right) d s\right\} \\
\leq & \underline{\lim _{k \rightarrow \infty}} \mathbb{E}_{n_{k}, \varepsilon}^{x, y} S_{t}^{n_{k}, \varepsilon}(f) \phi+\delta c_{1} \leq \varliminf_{k \rightarrow \infty} \mathbb{E}_{n_{k}, \varepsilon}^{x, y} \phi S_{t^{\prime}}^{n_{k}, \varepsilon}(f)+\delta c_{1} \\
\leq & \varliminf_{k \rightarrow \infty} \mathbb{E}_{n_{k}, \varepsilon}^{x, y} \phi\left\{f \circ \rho_{t}\left(\xi_{t^{\prime} \wedge \tau}, \eta_{t^{\prime} \wedge \tau}\right)-\int_{0}^{t^{\prime}} \mathbf{1}_{\{s<\tau\}}\left[2(2-\varepsilon) f^{\prime \prime} \circ \rho\right.\right. \\
& \left.\left.\quad+((1-\varepsilon) J+\varepsilon C) f^{\prime} \circ \rho\right]\left(s, \xi_{s}, \eta_{s}\right) d s\right\}+2 \delta c_{1} \\
= & \mathbb{E}_{\varepsilon}^{x, y} \phi S_{t^{\prime}}^{\varepsilon}(f)+2 \delta c_{1} .
\end{aligned}
$$

Letting $\delta \rightarrow 0$, we conclude that

$$
\mathbb{E}_{\varepsilon}^{x, y} \phi S_{t}^{\varepsilon}(f) \leq \mathbb{E}_{\varepsilon}^{x, y} \phi S_{t^{\prime}}^{\varepsilon}(f) .
$$

Similarly, let

$$
S_{t}(f)=f \circ \rho_{t \wedge \tau}\left(\xi_{t \wedge \tau}, \eta_{t \wedge \tau}\right)-\int_{0}^{t \wedge \tau}\left[J f^{\prime} \circ \rho+4 f^{\prime \prime} \circ \rho\right]\left(s, \xi_{s}, \eta_{s}\right) d s .
$$

Then $S_{t}^{\varepsilon}(f) \rightarrow S_{t}(f)$ uniformly as $\varepsilon \rightarrow 0$. By (4.11) and then with a same discussion as in (4.10), we obtain that

$$
\mathbb{E}^{x, y} \phi S_{t}(f) \leq \mathbb{E}^{x, y} \phi S_{t^{\prime}}(f),
$$

for all $t>t^{\prime}$ and $\mathscr{F}_{t^{\prime}}$-measurable nonnegative $\phi \in C_{b}\left(M_{x}^{T} \times M_{y}^{T}\right)$. This means that $S_{t}(f)$ is a $\mathbb{P}^{x, y}$ supermartingale.

(c) Now, let $f \in C^{2}(\mathbb{R})$ with $f^{\prime} \geq 0$ be fixed. For any $N \geq 1$, let

$$
T_{N}:=\inf \left\{t \in[0, T]: \rho_{t}\left(\xi_{t}, \eta_{t}\right) \leq 1 / N\right\} .
$$

One has $T_{N} \rightarrow T_{0}$ as $N \rightarrow \infty$. Let us take $\tilde{f} \in C^{2}(\mathbb{R})$ such that $\tilde{f}^{\prime} \geq 0,\left.\tilde{f}^{\prime}\right|_{[0,1 /(2 N)]}=0$ and $\tilde{f}=f$ on $[1 / N, \infty)$. Let

$$
d N_{t}(f):=d f \circ \rho_{t}\left(\xi_{t}, \eta_{t}\right)-\left[J f^{\prime} \circ \rho+4 f^{\prime \prime} \circ \rho\right]\left(t, \xi_{t}, \eta_{t}\right) d t, N_{0}(f):=f \circ \rho_{0}(x, y) .
$$

Then due to the concrete choice of $\tilde{f}$, one has $N_{t \wedge T_{N} \wedge \tau}(f)=S_{t \wedge T_{N} \wedge \tau}(\tilde{f})$ and hence $N_{t \wedge T_{N} \wedge \tau}$ is a supermartingale with respect to $\mathbb{P}^{x, y}$. Letting $N \rightarrow \infty$, we conclude that $N_{t \wedge T_{0} \wedge \tau}(f)$ is also a $\mathbb{P}^{x, y}$ supermartingale. Now, choosing explicit $f$ leads to obtain 4.1). The rest part is similar to the proof [35, Theorem 2.1.1](c)(d), we omit it here.

When $U \neq 0$, by replacing $L_{t}(y)$ with $L_{t}(y)+U(t, x, y)$ and with a similar argument as above, we then complete the proof.

4.2. Transportation-cost inequalities. In this subsection, we apply coupling methods to get some transportation-cost inequalities.

We denote

$$
W_{p, t}(\mu, v)=\left(\inf _{\eta \in \mathscr{C}(\mu, v)} \int_{M \times M} \rho_{t}^{p}(x, y) d \eta(x, y)\right)^{1 / p},
$$

the Wasserstein distance associated to $p \geq 1$, where $\mathscr{C}(\mu, v)$ is the set of all probability measures on $M \times M$ with marginal $\mu, v \in \mathscr{P}(M)$ and $\mathscr{P}(M)$ is the space of all the probability measures on $M$. 
Our main task in this subsection is to prove the transportation-cost inequalities on the manifolds carrying geometric flows. In fact, these inequalities have already investigated by constructing horizontal diffusion processes, see [2]. Now we review them by using coupling methods. Note that when the metric is independent of $t$, the equivalence of (i) and (ii) is due to [32].

Theorem 4.2. Let $p \geq 1$ and $K \in C\left(\left[0, T_{c}\right)\right)$. Then the following assertions are equivalent to each other.

(i) The curvature condition (3.17) holds for the function $K$.

(ii) For any $x, y \in M$ and $0 \leq s \leq t<T_{c}$,

$$
W_{p, t}\left(\delta_{x} P_{s, t}, \delta_{y} P_{s, t}\right) \leq \rho_{s}(x, y) e^{-\int_{s}^{t} K(r) d r} .
$$

(ii') For any $v_{1}, v_{2} \in \mathscr{P}(M)$ and $0 \leq s \leq t<T_{c}$,

$$
W_{p, t}\left(v_{1} P_{s, t}, v_{2} P_{s, t}\right) \leq W_{p, s}\left(v_{1}, v_{2}\right) e^{-\int_{s}^{t} K(r) d r} .
$$

Proof. It is easy to see that (ii') and (ii) are equivalent. It suffices for us to show that "(i) $\Leftrightarrow$ (ii)". By using coupling process $\left(X_{t}, \tilde{X}_{t}\right)$ by parallel displacement in Theorem 4.1 with $U=0$, we obtain from (i) that

$$
W_{p, t}\left(\delta_{x} P_{s, t}, \delta_{y} P_{s, t}\right) \leq\left\{\mathbb{E}\left(\rho_{t}\left(X_{t}, \tilde{X}_{t}\right)^{p} \mid\left(X_{s}, \tilde{X}_{s}\right)=(x, y)\right)\right\}^{1 / p} \leq \rho_{s}(x, y) e^{-\int_{s}^{t} K(u) d u} .
$$

That is, (i) implies (ii). On the other hand, if (ii) holds, then letting $\Pi_{x, y}$ be the optimal coupling for $\delta_{x} P_{s, t}$ and $\delta_{y} P_{s, t}$ for the $L^{p}$-transportation cost for $f \in C^{1}(M)$ and $f$ is constant outside a compact set, we have

$$
\begin{aligned}
& \left|\nabla^{s} P_{s, t} f\right|_{s} \leq \lim _{y \rightarrow x} \frac{\int_{M \times M}\left|f\left(x^{\prime}\right)-f\left(y^{\prime}\right)\right| \Pi_{x, y}\left(d x^{\prime}, d y^{\prime}\right)}{\rho_{s}(x, y)} \\
\leq & \lim _{y \rightarrow x}\left[\int_{M \times M}\left(\frac{\left|f\left(x^{\prime}\right)-f\left(y^{\prime}\right)\right|}{\rho_{t}\left(x^{\prime}, y^{\prime}\right)}\right)^{p /(p-1)} \Pi_{x, y}\left(d x^{\prime}, d y^{\prime}\right)\right]^{(p-1) / p} \cdot \frac{W_{p, t}\left(\delta_{x} P_{s, t}, \delta_{y} P_{s, t}\right)}{\rho_{s}(x, y)} \\
\leq & e^{-\int_{s}^{t} K(u) d u}\left(P_{s, t}\left|\nabla^{t} f\right|_{t}^{p /(p-1)}\right)^{(p-1) / p}
\end{aligned}
$$

By Theorem 3.6"(ii) $\Rightarrow$ (i)", this implies (i).

Remark 4.3. Actually, coupling methods are powerful tools for investigating similar problems on other general spaces.

(i) Recently, transportation-cost inequalities on the path space of $L_{t}$-diffusion space have been investigated in [13] by construcing suitable coupling process.

(ii) We would like to indicate that the dimension-free Harnack inequalities estabalished in Theorem 3.9 also can be proved by using coupling methods. Indeed, the author investigated dimension-free Harnack inequalities for reflecting diffusion semigroups on time-varing manifold when $\partial M \neq \varnothing$, see [14] for details.

Acknowledgements The author was supported in part by NSFC (Grant No. C10915252), Zhejiang Provincial Natural Science Foundation of China (Grant No. GB16021090058) and the Natural Science Foundation of Zhejiang University of Technology (Grant No. 2014XZ011). 


\section{REFERENCES}

[1] Marc Arnaudon, Kolehe Abdoulaye Coulibaly, and Anton Thalmaier. Brownian motion with respect to a metric depending on time: definition, existence and applications to Ricci flow. C. R. Math. Acad. Sci. Paris, 346(13-14):773$778,2008$.

[2] Marc Arnaudon, Koléhè Abdoulaye Coulibaly, and Anton Thalmaier. Horizontal diffusion in $C^{1}$ path space. In Séminaire de Probabilités XLIII, volume 2006 of Lecture Notes in Math., pages 73-94. Springer, Berlin, 2011.

[3] D. Bakry. On Sobolev and logarithmic Sobolev inequalities for Markov semigroups. In New trends in stochastic analysis (Charingworth, 1994), pages 43-75. World Sci. Publ., River Edge, NJ, 1997.

[4] Dominique Bakry. L'hypercontractivité et son utilisation en théorie des semigroupes. In Lectures on probability theory (Saint-Flour, 1992), volume 1581 of Lecture Notes in Math., pages 1-114. Springer, Berlin, 1994.

[5] Dominique Bakry and Michel Émery. Hypercontractivité de semi-groupes de diffusion. C. R. Acad. Sci. Paris Sér. I Math., 299(15):775-778, 1984.

[6] Fabrice Baudoin, Michel Bonnefont, and Nicola Garofalo. A sub-Riemannian curvature-dimension inequality, volume doubling property and the Poincaré inequality. Math. Ann., 358(3-4):833-860, 2014.

[7] Jean-Michel Bismut. Large deviations and the Malliavin calculus, volume 45 of Progress in Mathematics. Birkhäuser Boston, Inc., Boston, MA, 1984.

[8] Mu-Fa Chen and Feng-Yu Wang. Application of coupling method to the first eigenvalue on manifold. Progr. Natur. Sci. (English Ed.), 5(2):227-229, 1995.

[9] Mu-Fa Chen and Feng-Yu Wang. Estimation of spectral gap for elliptic operators. Trans. Amer. Math. Soc., 349(3):1239-1267, 1997.

[10] Mu-Fa Chen and Feng-Yu Wang. General formula for lower bound of the first eigenvalue on Riemannian manifolds. Sci. China Ser. A, 40(4):384-394, 1997.

[11] Xin Chen, Li-Juan Cheng, and Jing Mao. A probabilistic method for gradient estimates of some geometric flows. Stochastic Process. Appl., 125(6):2295-2315, 2015.

[12] Li-Juan Cheng. An integration by parts formula on path space over manifolds carrying geometric flow. Sci. China Math., 58(7):1511-1522, 2015.

[13] Li-Juan Cheng. Transportation-cost inequalities on path spaces over manifolds carrying geometric flows. To appear in Bull. Sci. Math., 2015.

[14] Li-Juan Cheng and Zhang Kun. Reflecting diffusion processes on manifolds carrying geometric flow. To appear in J. Theoret. Probab., 2015.

[15] Koléhè A. Coulibaly-Pasquier. Brownian motion with respect to time-changing Riemannian metrics, applications to Ricci flow. Ann. Inst. Henri Poincaré Probab. Stat., 47(2):515-538, 2011.

[16] M. Cranston. Gradient estimates on manifolds using coupling. J. Funct. Anal., 99(1):110-124, 1991.

[17] Jozef Dodziuk. Maximum principle for parabolic inequalities and the heat flow on open manifolds. Indiana Univ. Math. J., 32(5):703-716, 1983.

[18] K. D. Elworthy and X.-M. Li. Formulae for the derivatives of heat semigroups. J. Funct. Anal., 125(1):252-286, 1994.

[19] Christine M. Guenther. The fundamental solution on manifolds with time-dependent metrics. J. Geom. Anal., 12(3):425-436, 2002.

[20] Robert Haslhofer and Aaron Naber. Weak solutions for the Ricci flow I. J. Eur. Math. Soc.(to appear), 2016.

[21] A. M. Il'in, A. S. Kalašnikov, and O. A. Oleĭnik. Second-order linear equations of parabolic type. Uspehi Mat. Nauk, 17(3 (105)):3-146, 1962.

[22] Wilfrid S. Kendall. Nonnegative Ricci curvature and the Brownian coupling property. Stochastics, 19(1-2):111-129, 1986.

[23] Kazumasa Kuwada. Convergence of time-inhomogeneous geodesic random walks and its application to coupling methods. Ann. Probab., 40(5):1945-1979, 2012.

[24] Kazumasa Kuwada and Robert Philipowski. Coupling of Brownian motions and Perelman's $\mathscr{L}$-functional. J. Funct. Anal., 260(9):2742-2766, 2011.

[25] Kazumasa Kuwada and Robert Philipowski. Non-explosion of diffusion processes on manifolds with time-dependent metric. Math. Z., 268(3-4):979-991, 2011.

[26] Xue-Mei Li. Strong p-completeness of stochastic differential equations and the existence of smooth flows on noncompact manifolds. Probab. Theory Related Fields, 100(4):485-511, 1994.

[27] Torgny Lindvall and L. C. G. Rogers. Coupling of multidimensional diffusions by reflection. Ann. Probab., 14(3):860872,1986

[28] Robert J. McCann and Peter M. Topping. Ricci flow, entropy and optimal transportation. Amer. J. Math., 132(3):711730, 2010.

[29] Anton Thalmaier. On the differentiation of heat semigroups and Poisson integrals. Stochastics Stochastics Rep., 61(34):297-321, 1997.

[30] Anton Thalmaier and Feng-Yu Wang. Gradient estimates for harmonic functions on regular domains in Riemannian manifolds. J. Funct. Anal., 155(1):109-124, 1998. 
[31] Neil S. Trudinger. Pointwise estimates and quasilinear parabolic equations. Comm. Pure Appl. Math., 21:205-226, 1968.

[32] Max-K. von Renesse and Karl-Theodor Sturm. Transport inequalities, gradient estimates, entropy, and Ricci curvature. Comm. Pure Appl. Math., 58(7):923-940, 2005.

[33] Feng-Yu Wang. Logarithmic Sobolev inequalities on noncompact Riemannian manifolds. Probab. Theory Related Fields, 109(3):417-424, 1997.

[34] Feng-Yu Wang. Equivalence of dimension-free Harnack inequality and curvature condition. Integral Equations Operator Theory, 48(4):547-552, 2004.

[35] Feng-Yu Wang. Functional Inequalities, Markov Semigroups and Spectral Theory. The Science Series of the Contemporary Elite Youth. Science Press, Beijing, 2005.

[36] Feng-Yu Wang. Second fundamental form and gradient of Neumann semigroups. J. Funct. Anal., 256(10):3461-3469, 2009.

[37] Feng-Yu Wang. Harnack inequalities on manifolds with boundary and applications. J. Math. Pures Appl. (9), 94(3):304-321, 2010.

[38] Feng-Yu Wang. Analysis for diffusion processes on Riemannian manifolds. Advanced Series on Statistical Science \& Applied Probability, 18. World Scientific Publishing Co. Pte. Ltd., Hackensack, NJ, 2014.

Li-Juan Cheng, Department of Applied Mathematics, Zhejiang University of Technology, Hangzhou 310023, The People's Republic of China.

E-mail address: chenglj@zjut.edu.cn 